3 Yifu Wang, ${ }^{1,2,3,4,5,16}$ Bingqian Guo, ${ }^{1,2,3,6,16}$ Yajie Guo, ${ }^{1,2,3,6,16}$ Nana Qi, ${ }^{1,2,3,6,16}$ Yufang

\section{A spatiotemporal steroidogenic regulatory network in human fetal adrenal}

\section{glands and gonads}

5 Cheng Su, ${ }^{1,2,3,4,5}$ Liying Zhang, ${ }^{11}$ Qingyun Zhang, ${ }^{1,2,3,4,5,12}$ Minxi Li, ${ }^{11}$ Jingling

6 Liao, ${ }^{1,2,3,6}$ Yunkun Yan, ${ }^{1,2,3,4,5}$ Xingning Mao, ${ }^{1,2,3,6}$ Yanyu Zeng, ${ }^{1,2,3,6}$ Jinghang

7 Jiang, ${ }^{1,2,3,13}$ Zhongyuan Chen, ${ }^{1,2,3,6}$ Yi Guo, ${ }^{1,2,3,13}$ Shuai Gao, ${ }^{14}$ Jiwen Cheng, ${ }^{1,2,3,4,5}$

8 Yonghua Jiang, ${ }^{1,2,3,6,15,{ }^{*}}$ Zengnan Mo, ${ }^{1,2,3,4,5,17, *}$

9

4 Lv, ${ }^{1,2,3,4,5,16} \mathrm{Yu} \mathrm{Ye},{ }^{1,2,3,4,5,7,8}$ Yan Huang, ${ }^{9}$ Xinyang Long, ${ }^{1,2,3,10}$ Hongfei Chen, ${ }^{9}$

${ }^{1}$ Center for Genomic and Personalized Medicine, Guangxi Medical University, Nanning, Guangxi 530021, China.

${ }^{2}$ Guangxi Collaborative Innovation Center for Genomic and Personalized Medicine, Nanning, Guangxi 530021, China.

${ }^{3}$ Guangxi Key Laboratory for Genomic and Personalized Medicine, Guangxi Medical University, Guangxi 530021, China

${ }^{4}$ Institute of Urology and Nephrology, the First Affiliated Hospital of Guangxi

Medical University, Nanning, Guangxi 530021, China.

${ }^{5}$ Department of Urology, the First Affiliated Hospital of Guangxi Medical University, Nanning, Guangxi 530021, China.

${ }^{6}$ Guangxi Collaborative Innovation Center for Biomedicine ( Guangxi-ASEAN Collaborative Innovation Center for Major Disease Prevention and Treatment ), Guangxi Medical University, Nanning, Guangxi 530021, China. 
$23{ }^{7}$ Department of Emergency, the Second Affiliated Hospital of Guangxi Medical

24 University, Nanning, Guangxi 530021, China.

$25 \quad{ }^{8}$ Department of Urology, the Second Affiliated Hospital of Guangxi Medical

26 University, Nanning, Guangxi 530021, China.

$27{ }^{9}$ Department of Obstetrics, the Second Affiliated Hospital of Guangxi Medical

28 University, Nanning, Guangxi 530021, China.

$29{ }^{10}$ School of Public Health of Guangxi Medical University, Nanning, Guangxi 530021,

30 China.

$31{ }^{11}$ Department of Gynecology, the Second Affiliated Hospital of Guangxi Medical

32 University, Nanning, Guangxi 530021, China.

$33{ }^{12}$ Department of Urology, the Affiliated Tumor Hospital of Guangxi Medical

34 University, Nanning, Guangxi 530021, China.

$35{ }^{13}$ Department of Gynecology and Obstetrics, the First Affiliated Hospital of Guangxi

36 Medical University, Nanning, Guangxi 530021, China.

$37{ }^{14}$ College of Animal Science and Technology, China Agricultural University, Beijing

38 100083, China.

$39{ }^{15}$ Life Sciences Institute, Guangxi Medical University, Nanning, Guangxi 530021,

40 China

$41{ }^{16}$ This authors contributed equally

$42 \quad{ }^{17}$ Lead Contact

$43 \quad{ }^{*}$ Correspondance: jiangyonghua@stu.gxmu.edu.cn (Y.J.), mozengnan@gxmu.edu.cn $44 \quad(\mathrm{Z} . \mathrm{M})$ 
45

46

47

48

49

50

51

52

53

54

55

56

57

58

59

60

\section{Abstract}

Human fetal adrenal glands produce substantial amounts of dehydroepiandrosterone (DHEA), which is one of the most important precursors of sex hormones. However, the underlying biological mechanism remains largely unknown. Herein, we sequenced human fetal adrenal glands and gonads from 7 to $14 \mathrm{GW}$ via the $10 \times$ Genomics single-cell transcriptome techniques and reconstructed their location information by Spatial Transcriptome, conducted COOL-seq for the $M C 2 R+$ inner zone steroidogenic cells during the time window of sex differentiation $(8-12 \mathrm{GW})$. We found that relative to gonads, adrenal glands begin to synthesize steroids early. The coordination among steroidogenic cells and multiple nonsteroidogenic cells promotes adrenal cortex construction and steroid synthesis. Notably, during the time window of sex differentiation (8-12 GW), key enzyme gene expression shifts to accelerate DHEA synthesis in males and cortisol synthesis in females. Furthermore, high SST+ expressions in the adrenal gland and testis amplify androgen synthesis in males. Our research highlights the robustness of the action of fetal adrenal glands on gonads to modify the process of sexual differentiation. 


\section{Introduction}

Human fetal adrenal glands are huge endocrine organs composed of a definitive zone (DZ), a fetal zone (FZ) (approximately 75\%), and a transition zone (TZ) (after 14 gestational weeks $[\mathrm{GW}]$ ) that produce huge amounts of dehydroepiandrosterone (DHEA) and cortisol(Ishimoto \& Jaffe, 2011, Melau, Nielsen et al., 2019). DHEA is the most prominent precursor for sex hormone(Melau et al., 2019).. DHEA is clearly the main source of placenta estradiol (E2), which plays a critical role in regulating the development and maturation of fetal and maternal-fetal interface(Ishimoto \& Jaffe, 2011, Miller \& Auchus, 2019). In testis or ovary, DHEA is processed to become a different subtype of testosterone or estrogen, which drives sexual differentiation in organs(Asby, Arlt et al., 2009, Ishimoto \& Jaffe, 2011, Melau et al., 2019).

The human fetal gonads are "bipotential" before 8 GW(Rotgers, Jorgensen et al., 2018).. Gonads regulate the sexual differentiation of external reproductive organs by synthesizing sex hormones in a dose-dependent manner(Christou \& Tigas, 2018, Rotgers et al., 2018, Scott, Mason et al., 2009). Extensive evidence shows that the dysfunction of fetal adrenal glands leads to reproduction-related diseases, such as polycystic ovary syndrome(Goodarzi, Carmina et al., 2015), impaired spermatogenesis, and testicular atrophy(Scott et al., 2009). If the malfunction in adrenal glands is caused by mutations in various genes, such as CYP21A2, CYP11B1, and $P O R$, and when adrenal dysfunction occurs before the window of sexual differentiation, the malfunction may cause serious virilization or feminization(Asby et al., 2009, Miller \& Auchus, 2011). On account of DHEA circulation in fetal adrenal 
glands and gonads, we hypothesized that adrenal glands and the steroidogenesis regulatory network between adrenal glands and gonads may play a critical role in initial sexual development during the time window of sex differentiation.

However, investigating the functions of human fetal adrenal glands is difficult for two reasons. First, the origin of circulating DHEA and adrenal-derived androgens in humans and nonhuman primates is distinct from that in other mammalian species(Abbott \& Bird, 2009, Ishimoto \& Jaffe, 2011). Second, adrenal glands are a complex structure with various cellular component organs consisting of numerous heterogeneous cell types; most cortical cells originate from the mesoderm, neurocytes from the ectoderm(Dong, Yang et al., 2020, Furlan, Dyachuk et al., 2017), and germ cells from the yolk sac(Upadhyay \& Zamboni, 1982). The precise composition of tissue structures and functions of fetal adrenal glandsare unclear.

The recent development of single-cell sequencing technology has allowed the determination of complicated landscape of cell biology in the tissue(Bian, Gong et al., 2020, Dong et al., 2020, Furlan et al., 2017, Li, Dong et al., 2017, Tang, Barbacioru et al., 2009). Through the joint use of sc-RNA sequencing, Spatial Transcriptomics and multi-omics sequencing technology (COOL-seq)(Guo, Li et al., 2017, Satija, Farrell et al., 2015, Stahl, Salmen et al., 2016), we can make a comprehensive and thorough analysis of the spatial and temporal information within the organization from the dimension of multi-omics. In this study, we draw a fine map of the adrenal glands and gonads of 7-14 GW human fetal, which provide an unprecedented resource to understand the complexity of adrenal glands and shed light on the action of fetal 
bioRxiv preprint doi: https://doi.org/10.1101/2021.12.22.473776; this version posted December 24, 2021. The copyright holder for this preprint (which was not certified by peer review) is the author/funder. All rights reserved. No reuse allowed without permission.

105 adrenal glands and gonads to modify the process of sexual differentiation. 


\section{Results}

\section{Expression Programs of Cell Lineages in Fetal Adrenal Glands}

To generate a comprehensive landscape of steroid hormones in fetal adrenal glands and gonads spanning the window of sexual differentiation, adrenal glands and gonads (7-14 GW) samples were collected. The samples were digested and subjected to single-cell transcriptome sequencing by using the $10 \times$ Genomics system. Frozen sections from 8-9 GW fetal adrenal tissues were used for spatial transcriptome. The samples were classified into three stages: before $(<8 \mathrm{GW})$, within $(8-12 \mathrm{GW})$, and after the window of sexual differentiation (>12 GW)(Hanley \& Arlt, 2006) (Fig 1A). A total of 75,482 adrenal cells and 53,508 gonad cells passed the standard quality control. Filtered out erythrocytes were retained for downstream analysis (Table EV1).

On the basis of $10 \times$ Genomics data, uniform manifold approximation and projection analysis (UMAP) revealed 12 main cluster groups (Fig 1B and C), including the cells that mainly perform steroidogenic functions that previous studies defined as steroidogenic cells (CYP17A1+)(Ishimoto \& Jaffe, 2011), as well as other cells, such as neurocytes (CHGA + chromaffin cells, CARTPT + sympathoblasts, S100B+ Schwann cells)(Furlan et al., 2017) and immune cells (CD68+ macrophage, NKG7+ NK/T cells)(Bian et al., 2020) (Fig 1C; Table EV2).

\section{Spatiotemporal transcriptome characteristics of steroidogenic cells}

Steroidogenic cells, classically defined by $C Y P 17 A 1$, are a manifestation of adrenal 
function for steroid biosynthesis(Miller \& Auchus, 2011). We performed an unsupervised analysis of the gene expression profiles of these cells. Eight subtypes of steroidogenic cells were identified (Fig 2A and B; Table EV3). The gene expression heat map showed that the relative expression levels of the genes known to be associated with cyst stem cells (VSNL1 and NOV in T3 and COL1A1 and RSPO3 in T4), proliferation (MKI67, TOP $2 A$ in T2), and steroidogenic metabolism genes (CYP11B1 and SULT2A1 mainly in T3 and T5) varied in the eight subtypes (Fig 2B). T2 had a high cell cycle score defined as proliferative cells (Fig EV1A). Gene ontology (GO) analysis revealed that chromosome segregation, organelle fission, and nuclear division were enriched in subtype T2 (Fig EV1C). NOV is a marker gene for DZ, it is highly expressed in T3; SULT2A1 and CYP11B1 were highly expressed in T1 and T5 which characterized as FZ (Fig 2B; Table EV3). Immunostaining showed that cells expressing $N O V, M C 2 R$ and $C Y P 17 A 1$ were sequentially distributed from DZ to FZ (Fig EV1B). The characteristics of T4 cells were similar to those of fibroblasts (Fig 2B; Table EV3) and the steroidogenic genes were limited, GO analysis revealed that extracellular matrix organization, extracellular structure organization, and axonogenesis were enriched in this subtype (Fig EV1C), indicating that T4 may be involved in forming the adrenal zone.

Spatial Transcriptome was used to reproduce the spatial positions of these types of steroidogenic cell(Stahl et al., 2016). The T1 and T5 steroidogenic cells were located in the FZ of the fetal adrenal; the $\mathrm{T} 3$ steroidogenic cells were mostly distributed in the outside of T1 and T5 steroidogenic cells, considered as the cells 
located in DZ. T4 steroidogenic cells, the progenitor cell as we defined, were only distributed in the peripheral location, which is under the cysts (Fig 2E and EV1D). In accordance with the classical subcapsular DZ to FZ centripetal differentiation model(Ishimoto \& Jaffe, 2011), pseudotime analysis revealed a developmental trajectory of steroidogenic cells from T4 to other subtypes (Fig 2C). With the differentiation and maturity of steroidogenic cells, the steroidogenic-related genes CYP17A1, CYP11B1 and CYP21A2 were highly expressed (Fig 2C and EV1E). In summary, the single cell RNA sequencing data combined with Spatial Transcriptome enables us to support the classical model of the central differentiation and maturation of fetal adrenal steroidogenic cells from under the cyst.

The dot plots showed that along with fetal development, the motility regulator LIMD2, the lipid transport mediators $N R 2 F 2$ and VIM, and pluripotent genes (e.g., GPC3, SFRP1, and LMCD1) were progressively downregulated. Moreover, steroidogenic regulation-related genes, such as $L R M D A, A L K A L 2$, and CYP21A2, were upregulated (Fig 2D). Steroidogenic cells with high expression of CYP17A1 and CYP11B1 were enriched in the innermost FZ while the cells high expresses CYP21A2 were highly outside of them (Fig EV1F). Cells high expressing HSD3B2 and CYP11B2 were in the outermost part of the fetal adrenal glands (Fig EV1F). The $M C 2 R$, encoding the protein of $\mathrm{ACTH}$ receptor, is widely expressed in the $\mathrm{FZ}$ and $\mathrm{TZ}$ (Fig EV1F). Subsequently, $M C 2 R$ was used as marker to sort the steroidogenic cells in inner zone. 
172

173

\section{Steroidogenic Cells in Adrenal Glands Exhibit Distinct Expression Profiles across}

\section{Different Sexes}

Same as previously reported(Goto, Piper Hanley et al., 2006, Melau et al., 2019), a crest of $H S D 3 B 2$ within the sexual differentiation window only in females (Fig 3A and B). The temporarily elevated $H S D 3 B 2$ was thought to increase cortisone production and suppress DHEA synthesis through the competitive consumption of common precursors(Goto et al., 2006, Melau et al., 2019). However, the expression of another key enzyme for producing cortisol, CYP21A2, was limited in this period (Fig 2D), while CYP11B1 were detected. CYP11B1 can not only produce cortisol, but also play a key role in the biosynthesis of 11-Oxygenated androgens, which are new highly bioactive androgen mainly synthesis in androgen (Davio, Woolcock et al., 2020, Pretorius, Arlt et al., 2017, Turcu, Rege et al., 2020). In order to explore whether sexual dimorphism exist in the epigenetic regulation of steroidogenic cells during the window of sexual differentiation, we conducted COOLseq(Guo et al., 2017) on the adrenal $M C 2 R+$ steroidogenic cells (Fig 1A). The DNA methylation and chromatin state in those steroidogenic cells were detected. Although there were no significant differences in overall methylation levels between male and female, we found that the epigenetic profiles of the enzyme genes related steroid metabolizing are different. The methylation levels of exons in $H S D 3 B 2$ and CYP11B1 were higher in males, while $C Y B 5 A$ was higher in females (Fig 3D). The chromatin accessibility of promoter regions is known to be highly associated with expression levels of the corresponding genes(Guo et al., 2017). CYP21A2, a key enzyme for 
194

producing cortisol was only can be accessed in female. Meanwhile, the CYB5A in male has more chromatin opening (Fig 3E). These results suggest that the steroidogenic cells in female are more likely to synthesize cortisol, on the other hand, most willing to synthesize DHEA in male.

Taken together, the data indicated the dynamic changes in the steroid synthesis function of the steroidogenic cells and revealed the sex-specific guiding mechanism in the window of sexual differentiation.

\section{Neurocytes in Fetal Adrenal Glands Participate in Steroid Regulation}

To explore whether the fetal adrenal neurocytes have steroidogenic functions, the neurocyte group was further divided into 15 distinct subtypes, including 4 chromaffin cell subtypes, 2 sympathoblast subtypes, and 3 Schwann cell precursor (SCP) subtypes (Fig 4A and B; Table EV4).

The chromaffin cells expressed the marker genes $C H G A$ and $P H O X 2 B$. In addition, the T2 chromaffin cells specifically expressed SRD5A1 and AKR1C2, and these cells may synthesize the active androgen dihydrotestosterone (DHT) through a "backdoor pathway" (Fig 4A-C)(Miller \& Auchus, 2019, Reisch, Taylor et al., 2019). there expression levels were confirmed by immunofluorescence staining (Fig 4E).

T2 SCPs expressed the neural progenitor marker genes $S 100 B$ and $S O X 10$, the myelination-associated gene $M P Z$, and the cell cycle-associated genes MKI67 and $T O P 2 A$, indicating that these cells are active proliferative cells (Fig 4B and EV2A; Table EV4). Trajectory analysis revealed that the chromaffin cells and sympathoblasts 
in humans originated from a cluster of SCPs(Dong et al., 2020) (Fig 4F and EV2B). This finding is highly consistent with that of previous research in mice(Furlan et al.,

218 2017), and two intermediate cell subtypes were found to serve as a continuous "bridge"

219 between the SCPs and mature neurocytes (Fig EV2B). These "bridge" cells expressed the SCP marker gene $H O X B 9$, the functional neurocyte genes $C H G A$ and $P H O X 2 B$, and the transient genes ASCL1 and DLL3 (Fig 4B and EV2B; Table EV4). Along with neurocyte development and maturation, the cell motility gene ACTC1 and the neurodevelopmental genes $A S C L 1, D L L 3, G P C 3$, and $H O X B 9$ (Furlan et al., 2017) were downregulated, whereas the cell mitosis-related genes $H S P A 1 B$ and $P R O X 1$ and the insulin mediator IRS2 were upregulated (Fig EV2C).

234 Somatostatin inhibits DHEA synthesis in primary fetal adrenal cells at low 235 concentrations and promotes DHEA synthesis at high concentrations due to the 236 inhibition of feminization (Fig 4I). The spatiotemporal expression of SST and the sex 237 differences of SST reactions in sexual differentiation warrant further research. 


\section{CD5L+ Macrophages Participate in Steroidogenesis}

240 The immune cells were further divided into 16 distinct subtypes (Fig EV3A-B; Table

241 EV5), which included 5 macrophage subtypes $(C D 68+C D 163+), 2$ NK/T cell 242 subtypes $(N K G 7+C D 3 D+)$, proliferative cell subtypes $(M K I 67+T O P 2 A+)$, and yolk 243 sac-derived myeloid-biased progenitors (YSMPs, defined by CD34+ MPO+ $244 A Z U 1+)($ Bian et al., 2020). Various subtypes of immune cells were found to express 245 lipid regulatory factors such as APOE and LIPA, and steroid biosynthesis factors, such as POMC, IGF1, and NR4A3. (Fig EV3C). Macrophages were the most abundant immune cells, and T4 macrophages especially expressed CD5L (Fig EV3A-C). T4

248 cells were found to be involved in cellular lipid catabolic processes and regulation of 249 plasma lipoprotein particle levels for lipid metabolism (Fig 3D). CD5L were co250 staining with the macrophage marker CD68 (Fig EV3E). For fetal development, $251 A C Y 3, C X 3 C R 1$, and HMGA2, which are associated with chemokines and 252 cell migration, were downregulated, whereas genes such as $R H O B$, which are 253 involved in mediating apoptosis in immune cells, were upregulated (Fig 3F). Notably, 254 the steroid regulator $N R 4 A 3$ was highly expressed during the sexual differentiation 255 window (Fig 3F) relative to the surge in DHEA secretion by the in vitro primary fetal 256 adrenal cell co-cultured with macrophages (Fig 3G and $\mathrm{H}$ ).

257 Macrophages were found to negatively regulate adrenal proliferation and 258 differentiation via the TGFb signaling pathway network(Riopel, Branchaud et al., 259 1989) (Fig EV4H-J). Spatial transcriptome revealed that macrophages marker by 

specificity of fetal adrenal immune cells for steroidogenic regulation.

\section{Regulatory Network of Cell-Cell Interactions in Fetal Adrenal Glands}

268 The microenvironment of steroidogenic function depends on the co-construction of various cells in fetal adrenal glands (Fig EV4A). CellChat analysis shows that the melanocortin signaling pathway acted as an autocrine pathway through the $P O M C$ $M C 2 R$ ligand receptor (Fig EV4B), as verified by Spatial transcriptome and immunofluorescence staining (Fig EV4C-D). NPY signaling pathway communication, especially between neurocytes and steroidogenic cells, is associated with cholesterol metabolism(Chen, Zhou et al., 2020) (Fig EV2G-H). The AGT signaling pathway is found between vascular myocytes and steroidogenic cells, suggesting a specific effect

277 responsive cells(Pahlavani, Kalupahana et al., 2017) (Fig EV4E-F). The AGT-

278 AGTR1 interaction can be demonstrated in Spatial transcriptome (Fig EV4G).

\section{Gonadal Non-reproductive Cells Exhibit Diverse Functions}

281 To investigate the role of adrenal steroids in gonadal sex hormone synthesis for 

gonads data (Fig 5A-C; Table EV6). The groups were subsequently annotated using

284 known gene markers, such as FOXL2 for granulosa cells, $A M H$ for Sertoli cells, INSL3 for Leydig cells, and ARX for somatic progenitor cells(Rotgers et al., 2018) (Fig 5B).

The cell interactions in gonadal somatic cells (Sertoli cells, Leydig cells, and granulosa cells) were explored by CellChat (Fig 5D). Sertoli cells can be mediated by the activin, AMH, and opioid signaling pathways to form testicular cords and provide a favorable environment for germ cell survival(Li et al., 2017, Meroni, Galardo et al., 2019). In addition, Sertoli cells can be regulated by the autocrine melanocortin signaling pathway network, which may stimulate and promote the response of Sertoli cells to sex steroid biosynthesis(Aluru \& Vijayan, 2008) (Fig 5D). Leydig cells were found to be regulated by $\mathrm{GH}$ signaling pathways to induce steroidogenesis (Fig feminization to some extent(Banerjee, Das et al., 2019). The granulosa cell interaction signaling pathway is mainly involved in oogenesis and folliculogenesis in fetal ovaries(Li et al., 2017, Zhang, Yan et al., 2018). The WNT signaling pathway

299 promotes granulosa cell differentiation and inhibits apoptosis; the KIT and BMP 300 signaling pathways interact with germ cells to promote oogenesis and folliculogenesis 301 and protect preantral follicles from apoptosis(Rotgers et al., 2018, Zhang et al., 2018) 302 (Fig 5D). As a result of immature ovarian granulosa cell development in this period, 303 the function of ovarian steroidogenic regulation by the KIT and FSH signaling 
pathways may not be reflected (Fig $5 \mathrm{C}$ and D).

Sex Difference in SST+ Cells Indicated that the Dose-dependent Effect of

\section{Somatostatin Plays a Role in Sexual Differentiation}

Interestingly, SST in gonads was specifically expressed in a cluster of cells (Fig 5A and B and Fig EV5A). SST can regulate gonadotropins and thus affect the levels of sex steroids in gonads(Nakamura, Otsuka et al., 2013). The number of $S S T+$ cells in males was approximately twice as much as that in females during the window of sexual differentiation (Fig EV5B). They also showed sexual differences, similar to that previously mentioned in mature adrenal neurocytes (Fig 4G). These sexual disparities were confirmed by FACS and immunofluorescence staining (Fig EV5C-D). GO analysis identified gland development, reproductive structure development, reproductive system development, and mesenchyme development (Fig EV5E). The CellChat analysis was indicated that the SST+ cells were contact with Sertoli cells by desmosomes (Fig EV5F), which confirmed by immunofluorescence staining showed that those two types of cells were adhere closely with DSC2 (Fig EV5G). Furthermore, the peak numbers of $S S T+$ cells coincided with the time of emergence of Leydig cells, thereby possibly inhibiting $\mathrm{GH}$ to prevent feminization(Adams, Otero-Corchon et al., 2015, Banerjee et al., 2019) (Fig 5C, D and EV5B-D). As mentioned above, somatostatin exerts dose-dependent effects on sex steroids (Fig 4I), and measurement of its levels in gonads revealed high doses in testis and low doses in ovary (Fig EV5B-D). A high dose determines the "anti- 
326 female" mechanism of somatostatin that promotes androgen biosynthesis in testis, in

327 contrast to the ovary.

328 To determine the potential steroidogenesis network of human fetal adrenal glands

329 and gonads, we summarized the results of this research: adrenal glands begin to

330 express steroidogenic-related enzyme genes at approximately $7 \mathrm{GW}$, much earlier

331 than testis, and testis expresses steroid patterns that support de novo or partial

332 testosterone synthesis. An $H S D 3 B 2$ expression peak was observed in females at 10

333 GW. Adrenal glands may synthesize small amounts of DHT. Nevertheless, the

334 expression of steroidogenic enzymes in ovaries is limited until 14 weeks (Fig EV6). 


\section{Discussion}

Temporarily elevated HSD3B2 was thought to consume pregnenolone by

synthesizing cortisol and then reducing DHEA output, thereby avoiding the masculinization of women(Goto et al., 2006, Melau et al., 2019). In our data,

HSD3B2 was high express in female while CYP21A2 was limit during the window of

341 sex different, high CYP21A2 expression was not observed even until $14 \mathrm{GW}$. On account of CYP21A2 is one of the key enzyme of cortisol synthesis (Goto et al., 2006,

343 Melau et al., 2019), our data didn't support previous guesses. Our hypothesis is that 344 the steroid end-product of HSD3B2 is probably 11-Oxygenated androgens and not 345 cortisol(Davio et al., 2020, Pretorius et al., 2017, Turcu et al., 2020). The few reported 346 that the role of 11-Oxygenated androgens play in the maternal - fetal cycle in placenta.

347 Whether the 11-Oxygenated androgens participated in sexual differentiation mediated 348 by fetal adrenal was remain unknown(O'Shaughnessy, Antignac et al., 2019). The 349 possibility that 11-Oxygenated androgens may prevent female fetus masculinization 350 cannot be excluded. Further research is warranted to clarify the underlying molecular 351 mechanisms.

A study in mice reported that the cellular origin from Schwann cell precursors is the

353 stem cell of adrenal medulla cells, which in turn could develop into chromaffin cells

354 or sympathoblasts. A group of intermediate cell features that expresses $\mathrm{Htr} 3 \mathrm{a}+$ and 355 Ascl1+, which are transient intermediate cells, is called as "bridge cells" (Furlan et al., 356 2017). Notably, in the present study, the trajectory of SCP was highly consistent with 
357 that of mouse. In the trajectory analysis map is a group of ASCL1+ intermediate cells

358 that act as a bridge between SCP and different chromaffin cells and sympathoblasts.

359 Our research suggested that, although the adrenal glands between humans and mice

360 are remarkably different, they can still be reliably studied neurocyte in mouse models.

361 Notably, a cluster of SRD5A1+ and AKR1C2+ chromaffin cells was found in fetal 362 adrenal glands. These cells may synthesize active DHT by converting An 363 (androsterone), a process called the androgen synthesis "backdoor" pathway, which 364 corresponds to the conversion of testosterone to DHT via the "classic" 365 pathway(Miller \& Auchus, 2019, O'Shaughnessy et al., 2019, Reisch et al., 2019). 366 DHT is considered to be a highly bioactive androgen and reportedly promotes the 367 development and maturation of the nervous system(Starka, Duskova et al., 2015, 368 Venkatesh \& Monje, 2017). Thus, we surmise that DHT synthesis may promote the 369 maturation of adrenal neurocytes.

370 Surprisingly, SST expression was observed higher in male not only in adrenal 371 glands but also in gonads during the window of sexual differentiation. SST-encoding 372 somatostatin is a GH inhibitor and is reportedly associated with gonadotropin and the 373 development of femininity(Adams et al., 2015, Nakamura et al., 2013). The chromatin 374 accessibility of receptor genes SSTR2 was more highly in steroidogenic cells of males. 375 Thus, we speculate that SST expression is probably involved in steroid synthesis. Test 376 results of fetal adrenal organoids suggested that somatostatin inhibits DHEA synthesis 377 at low concentrations $(10 \mathrm{nmol} / \mathrm{L})$. However, this inhibitory effect is reversed at high 378 concentrations $(>100 \mathrm{nmol} / \mathrm{L})$. Hence, we hypothesize that this dose-dependent 
reactivity of somatostatin causes different outcomes between males and females.

Females would be in a long-term inhibition, whereas the opposite is true for males

381 due to the increase in somatostatin dose with the suppression of feminized transcription factors that ultimately promotes androgen synthesis in adrenal glands and gonads. Although this effect has been noted previously(Adams et al., 2015, Banerjee et al., 2019, Nakamura et al., 2013), it has not been observed in other adrenal or gonad single-cell sequencing studies. Further investigations, such as studies of mice with Sst conditional knockout, are needed to confirm the underlying molecular mechanism.

A group of $C D 5 L+$ macrophages were found in fetal adrenal glands, which were reportedly mediates lipid biosynthesis in testis(Wang, Yosef et al., 2015). Primary adrenal cell culture test showed a sharp increase in DHEA synthesis in a co-culture with macrophages. This result indicated that macrophages are involved in steroidogenic regulation and synthesis in adrenal glands.

393 Our study of steroidogenesis in human fetal adrenal glands and gonads during sexual differentiation at a single-cell solution not only confirmed past results but also

395 identified novel cell populations. To sum up, these findings can be linked together to 396 form spatiotemporal regulation network, highlighting spatiotemporal differences in 397 the steroidogenic regulatory network in adrenal glands and gonads, which we propose 398 as a "signal and fuel" phenomenon. $S R Y$ gene expression is the signal of male 399 differentiation, whereas DHEA synthesis in adrenal glands is the fuel that promotes male fetal differentiation on the right track. Our analyses provide novel insights into 
401 cell crosstalk during sexual differentiation in human fetal adrenal glands and gonads.

402 This study will deepen our understanding of the complex regulatory mechanism of

403 human fetal sexual differentiation. 
404

405

406

407

408

409

410

411

412

413

414

415

416

417

418

419

420

421

422

423

424

425

\section{Materials and Methods}

\section{Human Samples and Quality Control}

Human fetal adrenal and gonad tissues were isolated from aborted fetuses following elective surgical termination of pregnancy at the Second Affiliated Hospital of Guangxi Medical University, China. Every donor for the study provided informed consent. Gestational age was validated by ultrasound for crown rump length and fetal limb length to obtain more precise information about the age of the fetuses as described previously(Napolitano, Dhami et al., 2014). The sex of the fetus can be determined by illustrations of external genitalia or PCR SRY gene detection. The study was approved by the ethics committee of the Second Affiliated Hospital of Guangxi Medical University (KY-0096).

\section{Preparation of Cell Suspension}

Fresh samples of human fetal adrenal glands or gonads were placed in RPMI 1640 medium (C11875500BT, Gibco), which contained 5\% fetal bovine serum (FBS;

SH30070.03, HyClone) and 1\% penicillin-streptomycin (15140-122, Gibco), and quickly transported to the laboratory on ice. Then, the fetal adrenal glands and gonads were separated under a stereomicroscope (Nikon)and washed with cold D-PBS (311425-CL, Wisent) and sliced into approximately $1-2 \mathrm{~mm}^{3}$ pieces. The tissues were transferred to digestion solution $[0.1 \mathrm{mg} / \mathrm{ml}$ Liberase TL (5401020001, Roche) and 1 $\mathrm{mg} / \mathrm{ml}$ DNase I (10104159001, Roche) in RPMI 1640], at $37^{\circ} \mathrm{C}$ with gentle shaking 
426

427

428

throughout (adrenal glands for $20 \mathrm{~min}$ and gonads for $30 \mathrm{~min}$ ), filtered through a 100 $\mu \mathrm{m}$ cell strainer (352360, Falcon), centrifuged and resuspended in $5 \mathrm{ml}$ of $1 \mathrm{X}$ red blood cell (RBC) lysis buffer (420301, BioLegend) for 5 min on ice. Then washed twice with D-PBS containing 1\% FBS, filtered through a $40 \mu \mathrm{m}$ cell strainer (352340, Falcon), centrifuged and resuspended in DPBS with 1\% FBS. The cell number and viability were assessed by Trypan blue (152 50-061, Gibco) staining and counting in a counting chamber (717805, Brand).

\section{Single-cell RNA-seq Library Preparation and Sequencing}

We followed the approach of our previous studies(Liao, Yu et al., 2020, Yu, Liao et al., 2019). Reverse transcription and library preparation were performed using the $10 \mathrm{x}$ Genomics Single Cell v3 kit following the 10x Genomics protocol. Briefly, we added the single-cell suspension, gel beads and partitioning oil to 10x Genomics Chromium chip B and ran the Chromium Controller. After water-in-oil generation, samples were transferred into a PCR tube, and reverse transcription was performed using a T100 Thermal Cycler (Bio-Rad). Then, cDNA purification and library preparation were performed as the user guide. cDNA libraries were sent to Genergy Biotech (Shanghai) and sequenced by NovaSeq 6000 (Illumina).

\section{Spatial transcriptome library preparation and sequencing}

Fetal adrenal gland tissue samples were embedded in optimal cutting temperature compound and stored at $-80{ }^{\circ} \mathrm{C}$ in a sealed container. The RNA Integrity Number of 
448 fetal adrenal gland tissue collected tissue sections should be $\geq 7$ before placing the

449 tissue sections on Visium Spatial slides. A temperature of cryostat setting of $-20^{\circ} \mathrm{C}$ for

450 blade and $-10^{\circ} \mathrm{C}$ for the specimen head was recommended. Tissue blocks were cut

451 into $10 \mu \mathrm{m}$ sections and processed the capture areas using the Visium Spatial Gene

452 Expression Kit (10x Genomics) according to the manufacturer's instructions. Fetal

453 adrenal gland tissue permeabilization condition was optimized using the Visium

454 Spatial Tissue Optimization Kit. Sections were stained with H\&E and imaged using

455 Nikon Eclipse Ti2 microscop magnification, then processed for spatial transcriptomics

456 by consulting the Visium Spatial Gene Reagent Guidelines Technical Note

457 (CG000239) for more information. After the library construction, Agilent

458 2100/LabChip GX Touch was used to detect the fragment length distribution of the

459 library. Also, q-PCR technique was used to accurately quantify the effective

460 concentration of the library. The effective concentration of the library aimed $>$

461 10nmol/L. The qualified library was sequenced on an Illumina Novaseq 6000

462 platform. Cycling condition(Sequencing base length) were set 28, 90 and 10 for

463 read1, read2 and read 3 ( i7 index ), respectively .

465 COOL-seq library preparation and sequencing

466 The library preparation of COOL-seq was according to previous reports by Tang's

467 lab(Guo et al., 2017). After in vitro methylation of the bulk $M C 2 R+$ cells nuclei with

468 M.CviPI as previous Tang's protocol(Guo et al., 2017), the genomic DNA released by

469 proteinase treatment was used to construct the COOL-seq library using a PBAT 
471 Briefly, the bulk cells genomic DNA was bisulfite converted using the MethylCode

472 Bisulfite Conversion Kit (Invitrogen) following the manufacturer's instructions. Then,

473 the purified DNAs were annealed using random nonamer primers with a 5'-biotin tag

474 (5'-Biotin-CTACACGACGCTCTTCCGATCTNNNNNNNNN-3') in the presence of

475 Klenow fragments ( $3^{\prime}-5^{\prime}$ exo-, New England Biolabs). Then, the primers were

476 digested by exonuclease I (NEB) and the DNA was purified using Agencourt Ampure

477 XP beads (Beckman Coulter). Dynabeads M280 (Invitrogen, streptavidin-coupled)

478 were then used to immobilize the newly synthesized biotin-tagged DNA strands, and

479 the original bisulfite-converted DNA templates were removed. Second DNA strands

480 were synthesized using Klenow fragments with random nonamer primers (5'-

481 AGACGTGTGCTCTTCCGATCTNNNNNNNNN-3'). After washing, the beads were

482 used to amplify libraries using 13 cycles of PCR with the Illumina Forward PE1.0

483 primer and Illumina Reverse indexed primer (New England Biolabs) in the presence

484 of Kapa HiFi HS DNA Polymerase (Kapa Biosystems). The amplified libraries were

485 purified with Agencourt Ampure XP beads twice and were assessed on the Fragment

486 Analyzer (Advanced Analytical Technologies). Finally, libraries were pooled

487 (quantified with qPCR) and sequenced on the Illumina HiSeq 2500 sequencer for 150

488 bp paired-end sequencing. 
491 DNA was extracted from tissue samples according to the kit instructions (DN10, 492 Aidlab). Fragments of the exons of SRY genes were amplified by CWBIO $2 *$ ES Tap 493 Master Mix (Dye) (CW0690M, CWBIO) with primers (forward 5'min; 50 cycles $\left(94.0^{\circ} \mathrm{C}, 30 \mathrm{sec} ; 60.0^{\circ} \mathrm{C}, 30 \mathrm{sec} ; 72.0^{\circ} \mathrm{C}, 30 \mathrm{sec}\right)$, and $72.0^{\circ} \mathrm{C}$ for $2 \mathrm{~min}$.

\section{Immunofluorescence Staining}

The following protocols were used for staining of the fetal adrenal glands or gonads:

501 paraffin-embedded, 4- $\mu \mathrm{m}$ formalin-fixed tissue sections were dewaxed in xylene and rehydrated with distilled water. The sections were treated with EDTA at $\mathrm{pH} 8.5$

509 slides were washed three times in PBS for $5 \mathrm{~min}$ each, and then, the nuclei were

510 stained with DAPI (4083S, CST). Fluorescence images were captured using a laser

511 scanning confocal microscope (TCS SP8, Laika Microscope System Shanghai

512 Trading Co., Ltd.) and then processed with ImageJ software (NIH). 


\section{Flow Cytometry Analysis and Cell Sorting}

515 The cells were suspended in PBS (containing 1\% BSA), then incubated with antibody

516 (CD68, 1:100, 14-0688-82, Invitrogen or MC2R, 1:200, NB100-93419, Novus) at

$51744^{\circ} \mathrm{C}$ for $30 \mathrm{~min} . \mathrm{CD} 68$ was used for sorting macrophages (Bian et al., 2020)then wash

518 twice with PBS containing 1\% BSA. Then, the cells were incubated with Alexa Fluor

519 594-conjugated donkey anti-mouse $\operatorname{IgG}\left(1: 500\right.$, ab150112, Abcam) at $4^{\circ} \mathrm{C}$ in the dark

520 for $30 \mathrm{~min}$, then wash twice with PBS containing 1\% BSA. The cells were then

521 resuspended in PBS containing 1\% BSA for FACS. All samples were loaded on a BD

522 FACS Melody for flow cytometry cell sorting. After sorting, CD68-positive cells were

523 used for cell coculture and MC2R-positive cells were used for multiple omics

524 research.

525 Fetal gonadal cells a were fixed with fixation buffer (420801, Biolegend) at room

526 temperature and in the dark for $20 \mathrm{~min}$, then washed twice with 1X Intracellular

527 Staining Perm Wash Buffer (421002, Biolegend). The cells were incubated with

528 antibody (SST, 1:100, MA5-16987, Invitrogen) at $4^{\circ} \mathrm{C}$ for 30 minand washtwice with

529 1X Intracellular Staining Perm Wash Buffer. Then, the cells were incubated with

530 Alexa Fluor 647-conjugated donkey anti-rat IgG antibodies (1:500, ab150155,

531 Abcam) at $4{ }^{\circ} \mathrm{C}$ in the dark for $30 \mathrm{~min}$ and wash twice and resuspended with $1 \mathrm{X}$

532 Intracellular Staining Perm Wash Buffer.. All samples were loaded on a BD C6 Plus

533 for flow cytometry analysis. 


\section{Cell Culture}

536 Firstly, primary cell populations isolated from fetal adrenal glands were cultured in in 537 6-well plates (3516, Corning), with $2 \mathrm{ml}$ of medium in RPMI 1640 with $10 \%$ FBS 538 (SH30070.03, HyClone), 1\% penicillin/streptomycin (15140-122, Gibco) and $2 \mathrm{mM}$ 539 L-glutamine (25030081, Gibco) at $37^{\circ} \mathrm{C}$ in a humidified $5 \% \mathrm{CO}_{2}$ atmosphere. After 7 540 days, expanded adherent cells were trypsinized and reseeded in DMEM/F12 541 supplemented with 10\% FBS, 5\% horse serum (16050-122, Gibco), $100 \mu \mathrm{g} / \mathrm{ml}$ 542 primocin (ant-pm-1, InvivoGen), $100 \mathrm{ng} / \mathrm{ml}$ recombinant human FGF2 (100-18C, 543 PeproTech), and 2 mM L-glutamine(Poli, Sarchielli et al., 2019). Primary cell 544 populations were stained with CYP17A1 and MC2R to confirm that they were 545 steroidogenic cells.

\section{Cell Stimulation and Coculture}

548 Steroidogenic fetal adrenal cells were incubated in cultivation medium in each well 549 with or without $100 \mathrm{nmol} / 1 \mathrm{SST}$ (hor-299-a, ProSprc-Tany) and 0.1mmol/1 cholesterol 550 (C3045, Sigma) intervention for 24 h(Damsteegt, Hassan et al., 2019). The same cells 551 separately were cultivated in standard cell culture medium as a control. Steroidogenic 552 fetal adrenal cells were cultured with different SST concentrations $(-,+10 \mathrm{nmol} / \mathrm{l}$, $553+100 \mathrm{nmol} / \mathrm{l},+200 \mathrm{nmol} / \mathrm{l})$ of intervention. Basal DHEA and testosterone $(\mathrm{T})$ were 554 measured in the supernatants of cell culture after $24 \mathrm{~h}$ of cultivation.

555 Macrophages and steroidogenic fetal adrenal cells were cocultured in 24-well 556 Transwell chambers (3422, Corning). Steroidogenic fetal adrenal cells were incubated 
557 in the upper chamber at $6 \times 10^{4}$ cells/well, and macrophages were inoculated in the

558 lower chamber at a density of $4 \times 10^{4}$ cells/well. The macrophage-free group was used

559 as a control. Basal DHEA was measured in the supernatants of cell culture after $24 \mathrm{~h}$

560 of cultivation. The concentrations of DHEA and $\mathrm{T}$ in cell culture supernatants were 561 detected by ELISAs.

562

563 ELISA

564 Quantification of hormones was measured by ELISA with following kits: DHEA 565 ELISA kit (KJ-0766, Jingsu Kejing Biological Technology) and testosterone ELISA 566 kit (KJ-0779, Jingsu Kejing Biological Technology), according to the manufacturers' 567 instructions.

570 Raw data were demultiplexed using the mkfastq application (Cell Ranger v3.1.0) to 571 generate Fastq files. Fastq files then ran with count application (Cell Ranger v3.1.0) 572 using default settings, which perform alignment (using STAR aligner, aligned to the 573 GRCh38 human reference genomic data), filtering and UMI counting. UMI count 574 tables were used for further analysis.

576 Visium spatial transcriptomics data processing

577 Reads were demultiplexed and mapped to the reference genome GRCh38 using the 578 Space Ranger software v.1.0.0 (10x Genomics). Count matrices were loaded into 
579 Seurat v.3.1.1 and for all subsequent data filtering, normalization, filtering, 580 dimensional reduction and visualization. Data normalization was performed on 581 independent tissue sections using the variance-stabilizing transformation method 582 implemented in the SCTransform function in Seurat.

\section{Processing of COOL-seq data}

585 Based on Tang's previous research(Guo et al., 2017), raw reads were trimmed to 586 remove the first 9 bases and to remove the adapter-contaminated and low-quality 587 reads uing Trim Galore (v0.3.3). The cleaned and QC-ensured reads were then aligned 588 to the in-silico bisulfite-converted human genome reference (hg19) using Bismark 589 (v0.7.6) with paired-end and non-directional mapping parameters. After paired-end 590 mapping, the unmapped reads were re-aligned to the same reference genome in 591 single-end mode. Duplicated reads from the PCR amplification step were identified 592 and removed by using their genomic coordinates under published SAMtools (v0.1.18)

593 following the "samtools rmdup" command (v0.1.18) parameter: "samtools rmdup" for 594 paired-end reads and "samtools rmdup -s" for single-end reads. The process about 595 quantification of WCG and GCH methylation level were according to the previous 596 Tang's research(Guo et al., 2017). The visualization of methylation and chromatin 597 accessibility were based on The Integrative Genomics Viewer (IGV, v2.5.x) software. 
600 The "Seurat" package (v.3.1.1)(Satija et al., 2015) was used as the first analytical

601 package. For 10x Genomics data, UMI count tables from both replicates from all fetal

602 adrenal gland and gonad samples were loaded into R using the 'Read10X' function,

603 and 'Seurat' objects were built from each sample. Each object was filtered and

604 normalized with default settings. Specifically, cells were retained only if they

605 contained $>200$ expressed genes and $<4,500$ genes and had $<15 \%$ reads mapped to

606 the mitochondrial genome. After the cell filtration of each object, we used the 'merge'

607 function from "Seurat" to combine the objects into two main objects according to the

608 source of organs: adrenal and gonad, for downstream analysis. Cells were normalized

609 to the total UMI read counts by the 'NormalizeData', 'FindVariableFeatures' and

610 'ScaleData' functions, guided by tutorial in http://satijalab.org/seurat/. Then, the

611 objects were explored with the view of molecular networks for removing the batch

612 effect by the 'RSCORE' function from the "RSCORE" package(Dong, Zhou et al.,

613 2019). Principal component analysis was performed by the 'RunPCA' function from

614 "Seurat", and the top 35 principal components were selected for UMAP analysis. The

615 UMAP(Becht, McInnes et al., 2018) analysis was performed by the 'RunUMAP'

616 function from "Seurat". Similar cells were clustered and detected using the Louvain

617 method for community detection by the 'FindNeighbors' function. Discrete clusters

618 from adrenal data and gonad data were detected using 'FindClusters' and annotated by

619 specific cell markers from the "cellmarker" database (http://bio-

620 bigdata.hrbmu.edu.cn/CellMarker/). The erythrocytes (HBB, $A L A S 2)$ were filtered out 
621 by the 'subset' function. After removal of erythrocytes, 75,482 adrenal cells and

62253,508 gonad cells were retained for downstream analysis.

623 The screened cells were reclustered using the same analytical parameters. Forty-

624 two discrete clusters from adrenal data and 25 from gonad data were detected. The

625 clusters were annotated on the basis of feature genes. Finally, the clusters were

626 regrouped into the main cell groups by definition and similarity.

627

628 Cell Cycle Analysis

629 As in our previous studies(Yu et al., 2019), cell cycle analysis was performed by the

630 "Seurat" package by using previously defined cell cycle genes. We calculated a 'cycle

631 score' for each cell based on the expression of cell cycle genes. Cells were considered

632 aperiodic if the cycle score was less than two; otherwise, they were considered

633 proliferating.

634

\section{Gene Ontology (GO) and KEGG Analysis}

636 To detect differences in gene function expression among cell clusters in single-cell

637 data, we generated GO gene sets using datasets from "Seurat" single-cell object

638 conversion. GO enrichment analysis was conducted using the "clusterProfiler"

639 package (V3.12.0)(Yu, Wang et al., 2012). Before analysis, we transferred gene names

640 from 'symbol' to 'entrezid' according to the "org.Hs.eg.db" package

641 (V3.8.2)(Carlson, 2019). Terms with a p-value $<0.05$ were considered and enriched 
642

643

644

645

646

647

648

649

650

651

652

654

655

656 as significant. Dot plots and gene-concept networks were illustrated using the “enrichplot” package (V1.4.0)(Guangchuang Yu, 2019).

\section{Developmental Pseudotime Analysis}

Detailed pseudotime for different cell types was performed using the "Dyno" package (v2.10.1)(Saelens, Cannoodt et al., 2019) following the guideline settings. The inference methods in Dyno were wrapped within "Docker" containers (available at https://methods.dynverse.org). First, the 'wrap_expression' function was used to generate Dyno objects by transforming 'Seurat' object counts and normalized expression data. Then, the Dyno objects were preset to a "start_id" as an initial state of the cell by the "add prior information" function. In addition, the "add_grouping" function was used to add the results of the cell definition from 'Seurat' before the trajectory analysis. Finally, the "PAGA tree" method(Wolf, Hamey et al., 2019) was selected for trajectory analysis based on the characteristics of our data. Visualization of trajectory analysis was shown by the 'plot_dimred' function. All trajectory analysis models were constructed in the correct biological context.

\section{Cell-Cell Interaction Network Analysis}

The network analysis of signal interactions between cells was performed using the “CellChat" package(Jin, Guerrero-Juarez et al., 2021). The CellChat data were generated from the previous 'Seurat' object content by the 'data.input' function. The 'idents' in the CellChat data were obtained by the cell labels in Seurat objects. 
664 'Secreted signaling' and 'cell-cell contact', two models of cell interaction, were pulled 665 out from 'CellChatDB' for our follow-up analysis by the 'CellChatDB.use' function.

666 In the subsequent interpretation of the "CellChat" analysis results, we considered the 667 biological background and ignored some unreasonable results in the gonad data.

668

669

670 Data availability

671 Processed and raw human single cells sequencing data are available via the Gene

672 Expression Omnibus (GEO) (GEO: GSE167860).

673 
674

675

676

677

678

679

680

681

682

683

684

685

686

687

688

689

690

691

692

693

694

695

\section{Acknowledgements}

We thank the donors for participating in this study. We thank The Second Affiliated of

Guangxi Medical University for support sample collection; H. Mi, L. Mo from The

First Affiliated Hospital of Guangxi Medical University, H. Bai, M. Wei, Z. Liu, D.

Peng, Y. Su, S. Yi,from Gynaecology and Obstetrics Hospital of Guangxi Zhuang

Autonomous Region, Q Meng, C. Huang, Y. Xie, D. Li, P. Wei from Guangxi

Medical University, X. Sun from The Third Affiliated Hospital of Guangzhou Medical

University for experimental help; This work was supported by grants from the

National Key Research and Development Program of China (2017YFC0908000), the

Natural Key Research and Development Project (2020YFA0113200), the Major

Project of Guangxi Innovation Driven (AA18118016), the Guangxi key Laboratory

for Genomic and Personalized Medicine (16-380-54, 17-259-45, 19-050-22, 19-185-

33, 20-065-33), and the Natural Science Foundation of China (81770759, 82060145,

31970814).

\section{Author Contributions}

Y.W. Y.J., and Z.M. contributed to the study design. B.G., N.Q., Y.G. and Y.L. mainly performed cell suspensions, 10x single cell RNA-seq Library preparation. Y.G., N.Q. and Z.C. in PCR analysis. B.G., Y.G., Y.W. and N.Q. in immunofluorescence staining and confocal imaging. Y.G. performed cell stimulation, coculture and hormone quantification in vitro experiment. B.G. together with N.Q performed flow cytometry analysis and cell sorting. Y.W., Z.C., J.J., Y.Y., C.S. and Q.Z. collected fetal samples 
696 and patient information. Y.H, H.C, L.Z, M.L, J.C provided clinical technical guidance.

697 Y.W., Y.Y, X.M contributed to bioinformatic analyses. Y.W and Y.J. contributed to

698 manuscript preparation. Z.M. conceived and directed the study, obtained funding,

699 revised the manuscript. All authors provided critical proposal and approved the final

700 version of the manuscript.

701

702 Conflict of interest

703 The authors declare that they have no conflict of interest.

704 
705

706

707

708

709

710

711

712

713

714

715

716

717

718

719

720

\section{References}

Abbott DH, Bird IM (2009) Nonhuman primates as models for human adrenal androgen production: function and dysfunction. Rev Endocr Metab Disord 10: 33-42

Adams JM, Otero-Corchon V, Hammond GL, Veldhuis JD, Qi N, Low MJ (2015) Somatostatin is essential for the sexual dimorphism of $\mathrm{GH}$ secretion, corticosteroid-binding globulin production, and corticosterone levels in mice. Endocrinology 156: 1052-65

Aluru N, Vijayan MM (2008) Molecular characterization, tissue-specific expression, and regulation of melanocortin 2 receptor in rainbow trout. Endocrinology 149: 4577-88

Asby DJ, Arlt W, Hanley NA (2009) The adrenal cortex and sexual differentiation during early human development. Rev Endocr Metab Disord 10: 43-9

Banerjee S, Das RK, Shapiro BH (2019) Feminization imprinted by developmental growth hormone. Mol Cell Endocrinol 479: 27-38

Becht E, McInnes L, Healy J, Dutertre CA, Kwok IWH, Ng LG, Ginhoux F, Newell EW (2018) Dimensionality reduction for visualizing single-cell data using UMAP. Nat Biotechnol

Bian Z, Gong Y, Huang T, Lee CZW, Bian L, Bai Z, Shi H, Zeng Y, Liu C, He J, Zhou J, Li X, Li Z, Ni Y, Ma C, Cui L, Zhang R, Chan JKY, Ng LG, Lan Y et al. (2020) Deciphering human macrophage development at single-cell resolution. Nature 582: 571-576

Carlson M (2019) org.Hs.eg.db: Genome Wide Annotation for Human. R package Version 3.8.2.

Chen F, Zhou Y, Yang K, Shen M, Wang Y (2020) NPY stimulates cholesterol synthesis acutely by activating the SREBP2-HMGCR pathway through the $Y 1$ and $Y 5$ receptors in murine hepatocytes. Life Sci262: 118478 
727 Christou MA, Tigas S (2018) Recovery of reproductive function following androgen abuse.

728 Curr Opin Endocrinol Diabetes Obes 25: 195-200

729 Colon E, Svechnikov KV, Carlsson-Skwirut C, Bang P, Soder O (2005) Stimulation of

730 steroidogenesis in immature rat Leydig cells evoked by interleukin-1alpha is potentiated by

731 growth hormone and insulin-like growth factors. Endocrinology 146: 221-30

732 Damsteegt EL, Hassan Z, Hewawasam NV, Sarnsamak K, Jones PM, Hauge-Evans AC

733 (2019) A Novel Role for Somatostatin in the Survival of Mouse Pancreatic Beta Cells. Cellular

734 physiology and biochemistry : international journal of experimental cellular physiology,

735 biochemistry, and pharmacology 52: 486-502

736 Davio A, Woolcock H, Nanba AT, Rege J, O'Day P, Ren J, Zhao L, Ebina H, Auchus R,

737 Rainey WE, Turcu AF (2020) Sex Differences in 11-Oxygenated Androgen Patterns Across

738 Adulthood. The Journal of clinical endocrinology and metabolism 105

739 Dong J, Zhou P, Wu Y, Wang W, Chen Y, Zhou X, Xie H, Gao Y, Lu J, Yang J, Zhang X, Wen

740 L, Fu W, Li T, Tang F (2019) Enhancing single-cell cellular state inference by incorporating

741 molecular network features.

742 Dong R, Yang R, Zhan Y, Lai HD, Ye CJ, Yao XY, Luo WQ, Cheng XM, Miao JJ, Wang JF,

743 Liu BH, Liu XQ, Xie LL, Li Y, Zhang M, Chen L, Song WC, Qian W, Gao WQ, Tang YH et al.

744 (2020) Single-Cell Characterization of Malignant Phenotypes and Developmental Trajectories

745 of Adrenal Neuroblastoma. Cancer Cel/38: 716-733 e6

746 Furlan A, Dyachuk V, Kastriti ME, Calvo-Enrique L, Abdo H, Hadjab S, Chontorotzea T,

747 Akkuratova N, Usoskin D, Kamenev D, Petersen J, Sunadome K, Memic F, Marklund U, Fried

748 K, Topilko P, Lallemend F, Kharchenko PV, Ernfors P, Adameyko I (2017) Multipotent 
749 peripheral glial cells generate neuroendocrine cells of the adrenal medulla. Science 357

750 Goodarzi MO, Carmina E, Azziz R (2015) DHEA, DHEAS and PCOS. J Steroid Biochem Mol

751 Biol 145: 213-25

752 Goto M, Piper Hanley K, Marcos J, Wood PJ, Wright S, Postle AD, Cameron IT, Mason JI,

753 Wilson DI, Hanley NA (2006) In humans, early cortisol biosynthesis provides a mechanism to

754 safeguard female sexual development. J Clin Invest 116: 953-60

755 Guangchuang Yu EH (2019) Enrichplot: Visualization of Functional Enrichment Result. R

756 package Version 1.6.1.

757 Guo F, Li L, Li J, Wu X, Hu B, Zhu P, Wen L, Tang F (2017) Single-cell multi-omics

758 sequencing of mouse early embryos and embryonic stem cells. Cell Res 27: 967-988

759 Guo F, Yan L, Guo H, Li L, Hu B, Zhao Y, Yong J, Hu Y, Wang X, Wei Y, Wang W, Li R, Yan

760 J, Zhi X, Zhang Y, Jin H, Zhang W, Hou Y, Zhu P, Li J et al. (2015) The Transcriptome and

761 DNA Methylome Landscapes of Human Primordial Germ Cells. Cell 161: 1437-52

762 Hanley NA, Arlt W (2006) The human fetal adrenal cortex and the window of sexual

763 differentiation. Trends Endocrinol Metab 17: 391-7

764 Ishimoto H, Jaffe RB (2011) Development and function of the human fetal adrenal cortex: a

765 key component in the feto-placental unit. Endocr Rev 32: 317-55

766 Jin S, Guerrero-Juarez CF, Zhang L, Chang I, Ramos R, Kuan CH, Myung P, Plikus MV, Nie

767 Q (2021) Inference and analysis of cell-cell communication using CellChat. Nat Commun 12:

$768 \quad 1088$

769 Li L, Dong J, Yan L, Yong J, Liu X, Hu Y, Fan X, Wu X, Guo H, Wang X, Zhu X, Li R, Yan J,

770 Wei Y, Zhao Y, Wang W, Ren Y, Yuan P, Yan Z, Hu B et al. (2017) Single-Cell RNA-Seq 
771 Analysis Maps Development of Human Germline Cells and Gonadal Niche Interactions. Cell

772

773

774

775

776

777

778

779

780

781

782

783

784

785

786

787

788 Nakamura E, Otsuka F, Inagaki K, Tsukamoto N, Ogura-Ochi K, Miyoshi T, Toma K, Takeda

789

790

791

792

Stem Cel/20: 858-873 e4

Liao J, Yu Z, Chen Y, Bao M, Zou C, Zhang H, Liu D, Li T, Zhang Q, Li J, Cheng J, Mo Z

(2020) Single-cell RNA sequencing of human kidney. Scientific data 7: 4

Lumbers ER, Pringle KG (2014) The Roles of the circulating renin-angiotensin-aldosterone

system in human pregnancy. Am J Physiol Regul Integr Comp Physio/306: R91-101

Melau C, Nielsen JE, Frederiksen H, Kilcoyne K, Perlman S, Lundvall L, Langhoff Thuesen L, Juul Hare K, Andersson AM, Mitchell RT, Juul A, Jørgensen A (2019) Characterization of

Human Adrenal Steroidogenesis During Fetal Development. The Journal of clinical

endocrinology and metabolism 104: 1802-1812

Meroni SB, Galardo MN, Rindone G, Gorga A, Riera MF, Cigorraga SB (2019) Molecular

Mechanisms and Signaling Pathways Involved in Sertoli Cell Proliferation. Frontiers in

\section{Endocrinology 10}

Miller WL, Auchus RJ (2011) The molecular biology, biochemistry, and physiology of human steroidogenesis and its disorders. Endocr Rev 32: 81-151

Miller WL, Auchus RJ (2019) The "backdoor pathway" of androgen synthesis in human male sexual development. PLoS Bio/ 17: e3000198

M, Makino H (2013) Involvement of bone morphogenetic protein activity in somatostatin

actions on ovarian steroidogenesis. J Steroid Biochem Mol Biol 134: 67-74

Napolitano R, Dhami J, Ohuma EO, loannou C, Conde-Agudelo A, Kennedy SH, Villar J,

Papageorghiou AT (2014) Pregnancy dating by fetal crown-rump length: a systematic review 
793

794

795

796

797

798

799

800

801

802

803

804

805

806

807

808

809

810

811

812

813

814

of charts. BJOG 121: 556-65

O'Shaughnessy PJ, Antignac JP, Le Bizec B, Morvan ML, Svechnikov K, Soder O, Savchuk I,

Monteiro A, Soffientini U, Johnston ZC, Bellingham M, Hough D, Walker N, Filis P, Fowler PA

(2019) Alternative (backdoor) androgen production and masculinization in the human fetus.

PLOS Biol 17: e3000002

Pahlavani M, Kalupahana NS, Ramalingam L, Moustaid-Moussa N (2017) Regulation and

Functions of the Renin-Angiotensin System in White and Brown Adipose Tissue. Compr

Physiol 7: 1137-1150

Poli G, Sarchielli E, Guasti D, Benvenuti S, Ballerini L, Mazzanti B, Armignacco R, Cantini G,

Lulli M, Chortis V, Arlt W, Romagnoli P, Vannelli GB, Mannelli M, Luconi M (2019) Human

fetal adrenal cells retain age-related stem- and endocrine-differentiation potential in culture.

FASEB J 33: 2263-2277

Pretorius E, Arlt W, Storbeck KH (2017) A new dawn for androgens: Novel lessons from 11-

oxygenated C19 steroids. Mol Cell Endocrinol 441: 76-85

Reisch N, Taylor AE, Nogueira EF, Asby DJ, Dhir V, Berry A, Krone N, Auchus RJ,

Shackleton CHL, Hanley NA, Arlt W (2019) Alternative pathway androgen biosynthesis and

human fetal female virilization. Proc Nat/ Acad Sci U S A 116: 22294-22299

Riopel L, Branchaud CL, Goodyer CG, Adkar V, Lefebvre Y (1989) Growth-inhibitory effect of

TGF-B on human fetal adrenal cells in primary monolayer culture. J Cell Physio/ 140: 233-8

Rotgers E, Jorgensen A, Yao HH (2018) At the Crossroads of Fate-Somatic Cell Lineage

Specification in the Fetal Gonad. Endocr Rev 39: 739-759

Saelens W, Cannoodt R, Todorov H, Saeys Y (2019) A comparison of single-cell trajectory 
817 cell gene expression data. Nat Biotechno/33: 495-502

818 Scott HM, Mason JI, Sharpe RM (2009) Steroidogenesis in the fetal testis and its

819 susceptibility to disruption by exogenous compounds. Endocr Rev 30: 883-925

820 Smallwood SA, Lee HJ, Angermueller C, Krueger F, Saadeh H, Peat J, Andrews SR, Stegle

821 O, Reik W, Kelsey G (2014) Single-cell genome-wide bisulfite sequencing for assessing

822 epigenetic heterogeneity. Nat Methods 11: 817-820

823 Stahl PL, Salmen F, Vickovic S, Lundmark A, Navarro JF, Magnusson J, Giacomello S, Asp

824 M, Westholm JO, Huss M, Mollbrink A, Linnarsson S, Codeluppi S, Borg A, Ponten F, Costea

825 PI, Sahlen P, Mulder J, Bergmann O, Lundeberg J et al. (2016) Visualization and analysis of

826 gene expression in tissue sections by spatial transcriptomics. Science 353: 78-82

827 Starka L, Duskova M, Hill M (2015) Dehydroepiandrosterone: a neuroactive steroid. J Steroid

828 Biochem Mol Biol 145: 254-60

829 Tang F, Barbacioru C, Wang Y, Nordman E, Lee C, Xu N, Wang X, Bodeau J, Tuch BB,

830 Siddiqui A, Lao K, Surani MA (2009) mRNA-Seq whole-transcriptome analysis of a single cell.

$831 \quad$ Nat Methods 6: 377-82

832 Turcu AF, Rege J, Auchus RJ, Rainey WE (2020) 11-Oxygenated androgens in health and

833 disease. Nat Rev Endocrinol 16: 284-296

834 Upadhyay S, Zamboni L (1982) Ectopic germ cells: natural model for the study of germ cell 835 sexual differentiation. Proc Natl Acad Sci U S A 79: 6584-8

836 Venkatesh H, Monje M (2017) Neuronal Activity in Ontogeny and Oncology. Trends Cancer 3: 
839 Horste G, Pawlak M, Kishi Y, Joller N, Karwacz K, Zhu C, Ordovas-Montanes M, Madi A,

840 Wortman I, Miyazaki T, Sobel RA, Park H et al. (2015) CD5L/AIM Regulates Lipid

841 Biosynthesis and Restrains Th17 Cell Pathogenicity. Cel/ 163: 1413-27

842 Wolf FA, Hamey FK, Plass M, Solana J, Dahlin JS, Gottgens B, Rajewsky N, Simon L, Theis

843 FJ (2019) PAGA: graph abstraction reconciles clustering with trajectory inference through a

844 topology preserving map of single cells. Genome Bio/20: 59

845 Yu G, Wang LG, Han Y, He QY (2012) clusterProfiler: an R package for comparing biological

846 themes among gene clusters. OMICS 16: 284-7

847 Yu Z, Liao J, Chen Y, Zou C, Zhang H, Cheng J, Liu D, Li T, Zhang Q, Li J, Yang X, Ye Y,

848 Huang Z, Long X, Yang R, Mo Z (2019) Single-Cell Transcriptomic Map of the Human and

849 Mouse Bladders. Journal of the American Society of Nephrology : JASN30: 2159-2176

850 Zhang Y, Yan Z, Qin Q, Nisenblat V, Chang HM, Yu Y, Wang T, Lu C, Yang M, Yang S, Yao

851 Y, Zhu X, Xia X, Dang Y, Ren Y, Yuan P, Li R, Liu P, Guo H, Han J et al. (2018)

852 Transcriptome Landscape of Human Folliculogenesis Reveals Oocyte and Granulosa Cell

853 Interactions. Mol Cel/72: 1021-1034 e4

854 
A

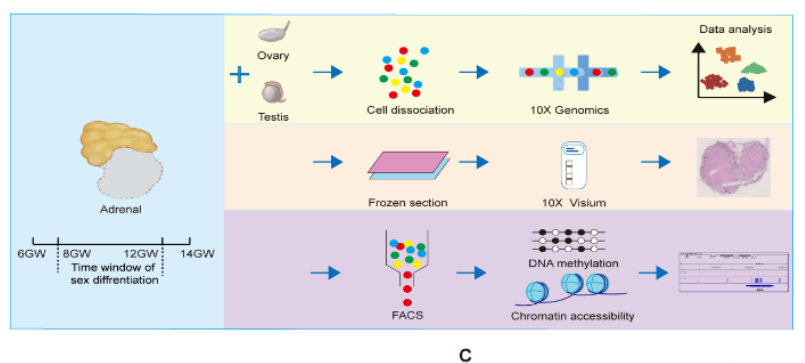

B

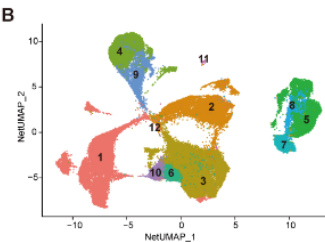

856 Figure 1. Global patterns of single-cell expression profiles of fetal adrenal glands.

857 A. Experimental schematic; 10 adrenal glands and 8 gonads in 10× Genomics; 2

858 adrenal glands in Spatial transcriptome, 10 adrenal glands in COOL-seq.

B. Uniform manifold approximation and projection analysis (UMAP) of the transcriptomes of fetal adrenal cells in $10 \times$ Genomics data $(n=75,482)$. The clusters were identified by marker genes, as shown in C and D.

C. Violin plot overview of the expression of selected marker genes by the fetal adrenal clusters. Detailed cell information and differentially expressed genes can be found in Table EV2. 
A

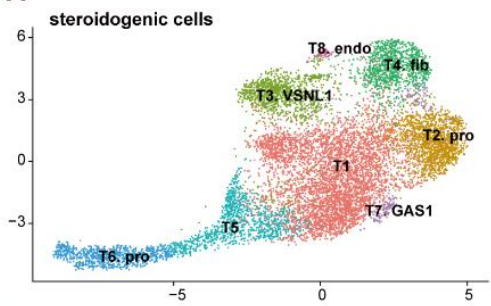

C

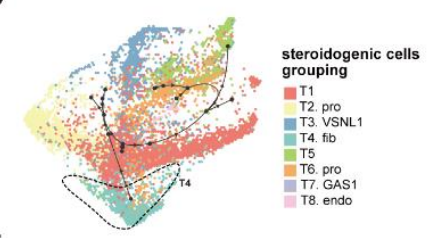

E
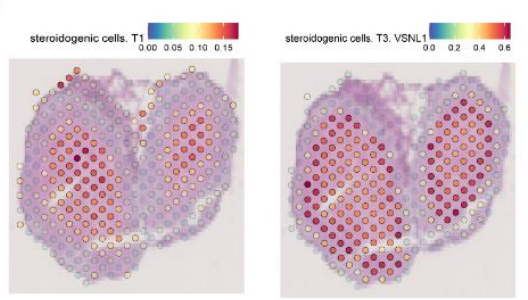

B

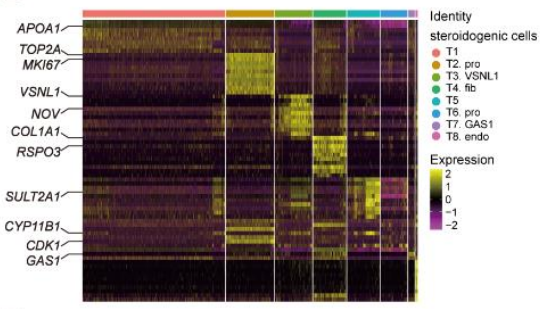

D
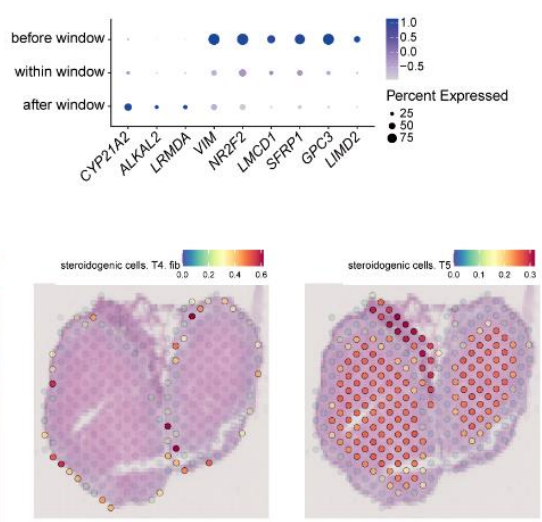

Figure 2. Dynamic gene expression patterns of fetal adrenal gland steroidogenic 868 cells.

869 A. Uniform manifold approximation and projection analysis visualization of professional steroidogenic cells for $10 \times$ Genomics data $(n=10,651)$.

B. Heat map of the top 10 differentially expressed genes (DEGs) between professional steroidogenic cell populations $(n=10,651)$. Color scale: yellow, high expression; purple, low expression. Detailed cell information and DEGs can be found in Table EV3.

C. Differentiation of trajectory of professional steroidogenic cells using Dyno. Arrow direction indicates the trajectory of cell differentiation.

D. Dot plots of differential gene expression of three-stage professional steroidogenic cell groups (before, within, and after sexual differentiation).

E. Visualization of the spatial transcriptome shows the location of T1, T3. VSNL1, T4. fib and T5 steroidogenic cells in 8GW fetal adrenals. 
A
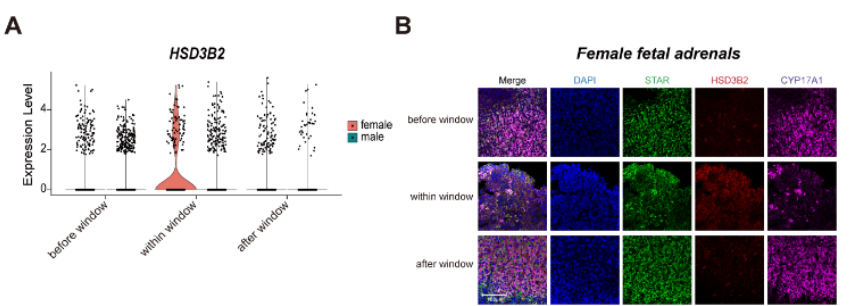

C

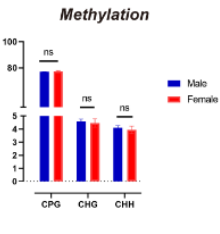

D

Methylation

E

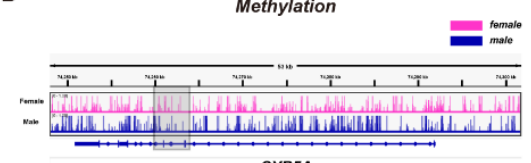

CYB5A

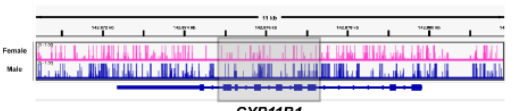

CYP11B1

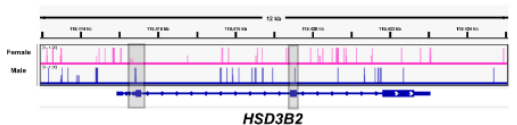

Chromatin accessibility
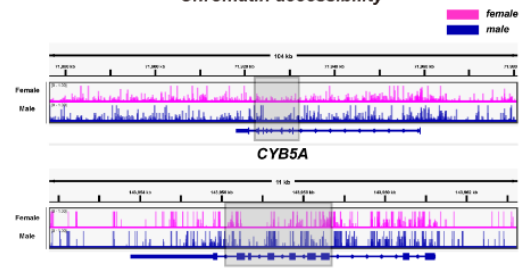

CYP11B1

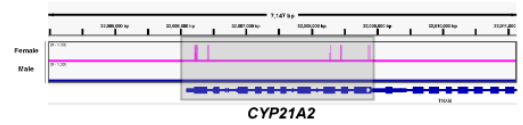

882

Figure 3. Dynamic gene expression patterns of fetal adrenal gland steroidogenic

884 cells.

885 A. Violin plots of $H S D 3 B 2$ gene expression patterns of female and male fetuses

886 within the window of sexual differentiation.

B. Immunofluorescence staining of StAR (green), HSD3B2 (red), and CYP17A1

888

(purple) in the female fetal adrenal glands spanning the window of sexual

889 differentiation. Scale bar, $20 \mu \mathrm{m} . \mathrm{n}=3$.

890

C. The overall methylation levels between male and female in COOL-seq.

891

D. The landscape of methylation site in CYB5A, HSD3B2, CYP11B1, CYP17A1 of

892 human fetal adrenal $M C 2 R+$ steroidogenic cells (female, purple; male, bule).

893

E. The landscape of chromatin accessibility site in $C Y B 5 A, C Y P 11 B 1, C Y P 17 A 1$, $C Y P 21 A 2$ of human fetal adrenal $M C 2 R+$ steroidogenic cells (female, purple; male, bule). 
A

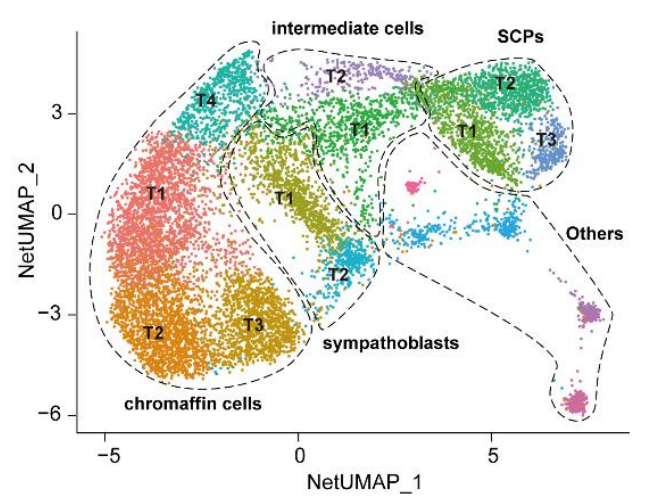

C
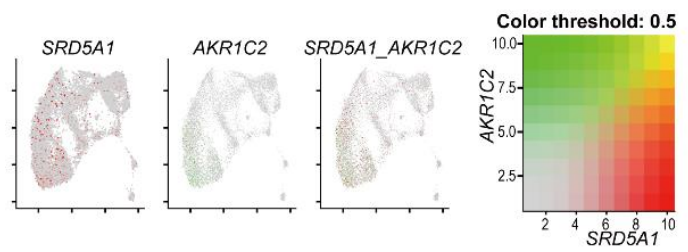

$\mathbf{E}$
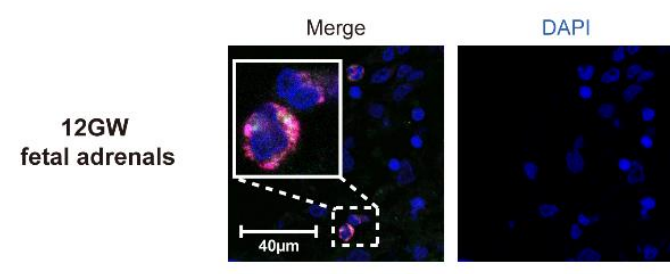

$\mathbf{F}$

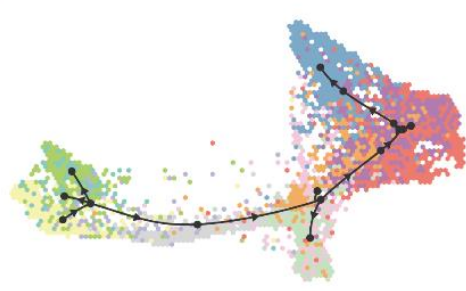

grouping

III chromaffin cells. T1 chromaffin cells. T2. backdor Chromaffin cells. T3. pro SCPS. T1
SCPS. T2. pro SCPS. T3. lipo - chromaffin cells. T4
intermediate cells. $T$. intermediate cells. T1 intermediate ells. $T 2$
sympathoblasts $T 1$
s. $T$. sympathoblasts. T1

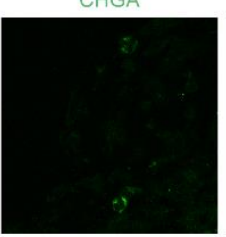

AKR1C2

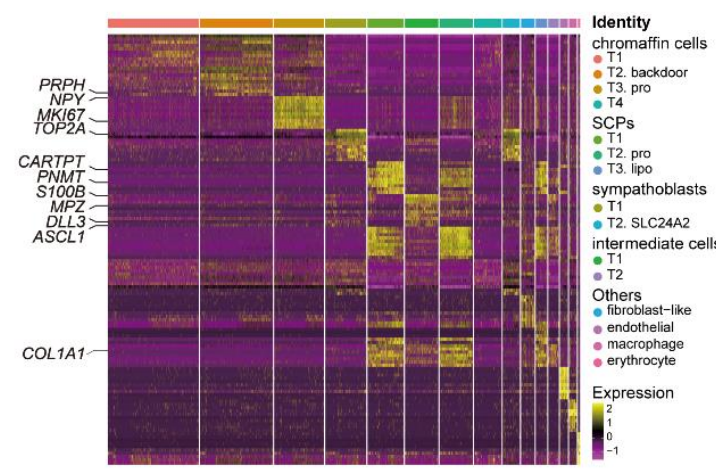

D

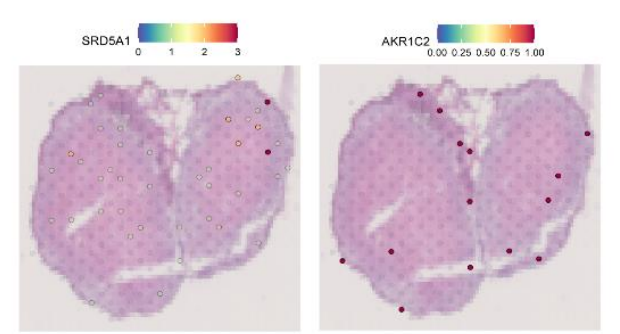

G
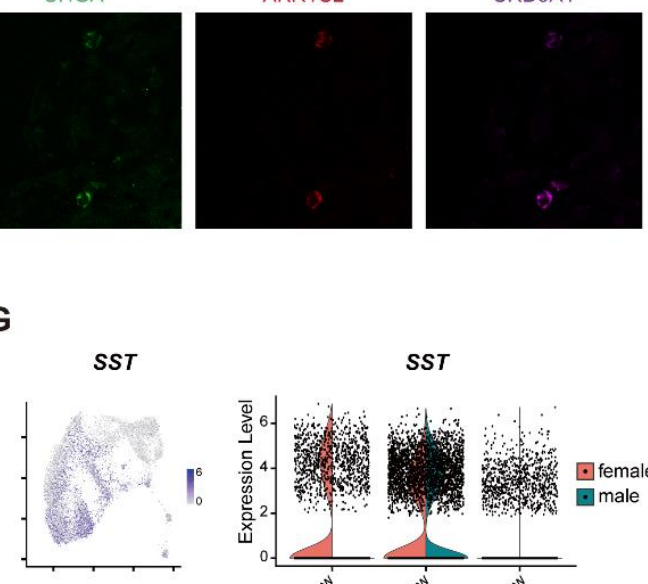

H
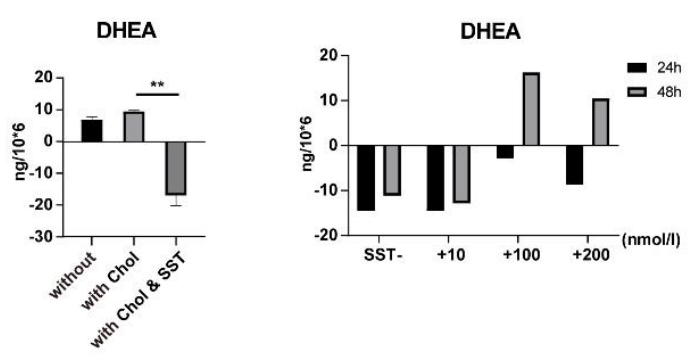

Figure 4. A landscape and characteristics of fetal adrenal gland neurocytes.

A. Uniform manifold approximation and projection analysis visualization of adrenal neurocytes for $10 \times$ Genomics data $(n=10,812)$. 
B. Heat map of the top 10 differentially expressed genes (DEGs) between adrenal neurocyte populations. Detailed cell information and DEGs can be found in Table EV4.

C. Expression patterns of $S R D 5 A 1$ and $A K R I C 2$ exhibited by feature plot visualization, which are key enzymes of the DHT "backdoor pathway". A gradient of gray, red, or green indicates low to high expression, and yellow indicates coexpression.

D. Visualization of the spatial transcriptome shows the locations which high express SRD5A1 and AKRIC2 in 8GW fetal adrenals.

E. Immunofluorescence staining of SRD5A1 (purple), AKR1C2 (red), and CHGA (green) in fetal adrenal tissues. Scale bar, $20 \mu \mathrm{m}$.

F. Differentiation of trajectory of professional steroidogenic cells using Dyno. Arrow direction indicates the trajectory of cell differentiation.

G. Feature plot visualization of SST in fetal adrenal neurocyte data, mainly expression in mature neurocytes (chromaffin cells and sympathoblasts). Violin plot of SST expression with gender differences.

H. ELISA of DHEA levels in the supernatant of the in vitro cultured fetal adrenal cells with or without somatostatin $(100 \mathrm{nmol} / \mathrm{l})$ and cholesterol $(0.1 \mathrm{mmol} / \mathrm{l})$ intervention. Chol, cholesterol; SST, somatostatin.

I. ELISA of DHEA in the supernatant of the in vitro cultured fetal adrenal cells

923 J. The landscape of chromatin accessibility site in SSTR2, of human fetal adrenal MC2R+ steroidogenic cells (female, purple; male, bule). 
A

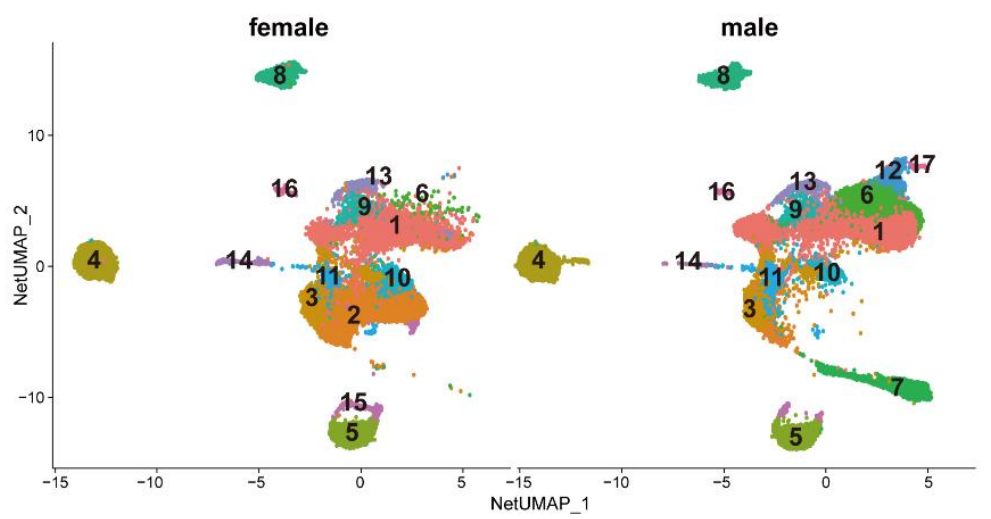

1. fibroblast

2 - granulosa cells

3 - neurosecretory cells

4 - macrophage

$5 \cdot$ germ cells

6 - somatic progenitor cells

7 - Sertoli cells

8 - vascular endothelial

9 - mesonephric cells

10 - urothelial cells

11- SST+ cells

12. Leydig cells

13- myocyte

14- podocyte-like

15- germ cells. meiosis

16. chromaffin cells

17- adrenal-like

B

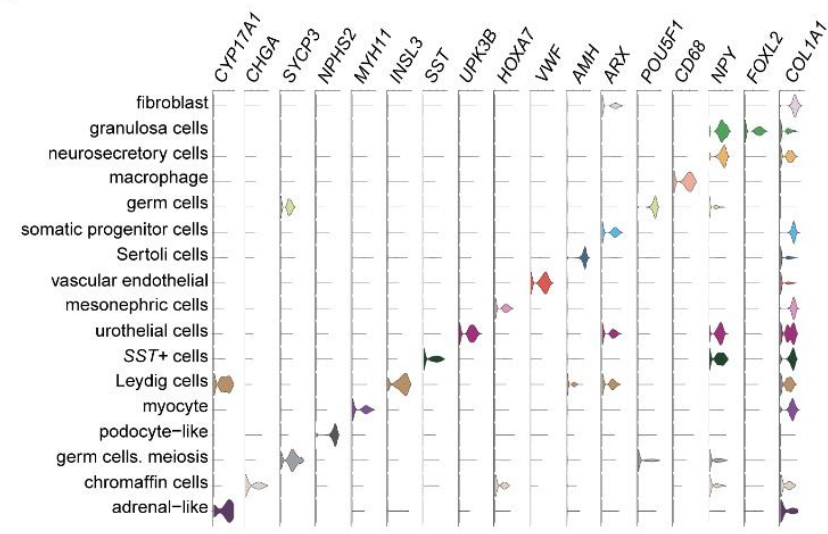

C

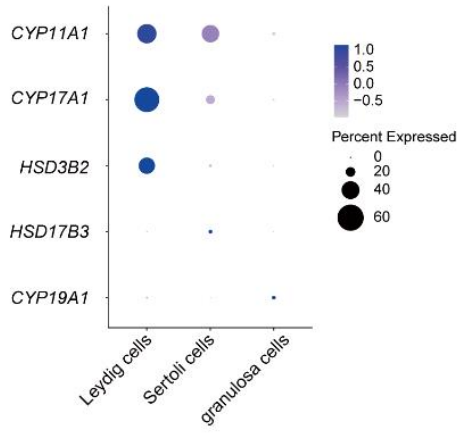

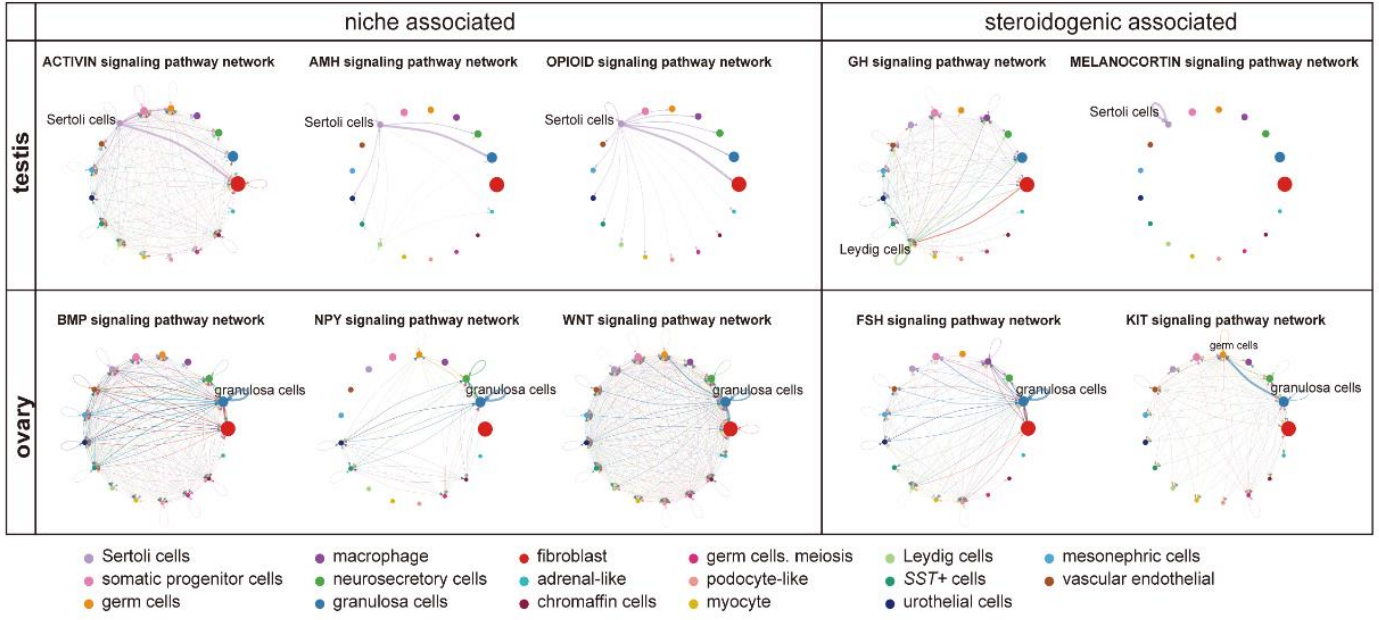

927 Figure 5. Transcriptomic landscape of human fetal gonads.

928 A. Uniform manifold approximation and projection analysis of the transcriptomes of all-stage fetal gonadal cells split by gender $(n=53,508)$. 
930 B. Violin plot overview of the expression of selected marker genes by gonad clusters.

931 Detailed cell information and differentially expressed genes can be found in $932 \quad$ Table EV6.

933 C. Dot plot showing the expression of key enzymes for sex hormone biosynthesis 934 (CYP11A1, CYP17A1, HSD3B2, HSD17B3, and CYP19A1) in different gonadal $935 \quad$ somatic cells.

936 D. CellChat analysis showing the cell-cell interaction of gonadal somatic cells in the 937 testis (up). The signal interaction pathway networks are divided into two 938 functions: niche-associated (activin, AMH, opioid) and steroidogenic-associated 939 (GH, melanocortin). The cell-cell interaction of gonadal somatic cells in the 940 ovary (below). The signal interaction pathway networks are divided into two 941 functions: niche-associated (BMP, NPY, WNT) and steroidogenic-associated 942 (FSH, KIT).

943 
A

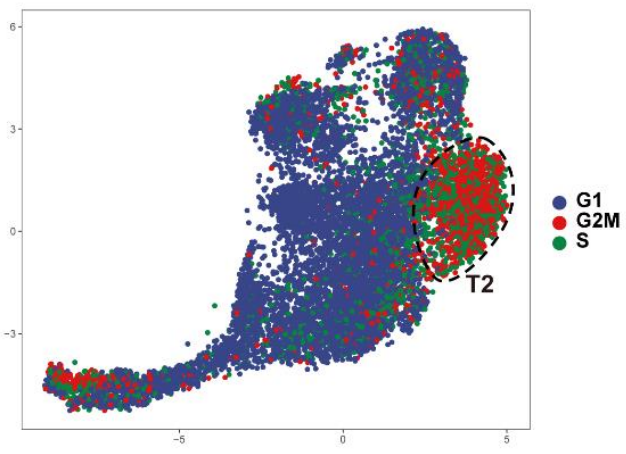

C
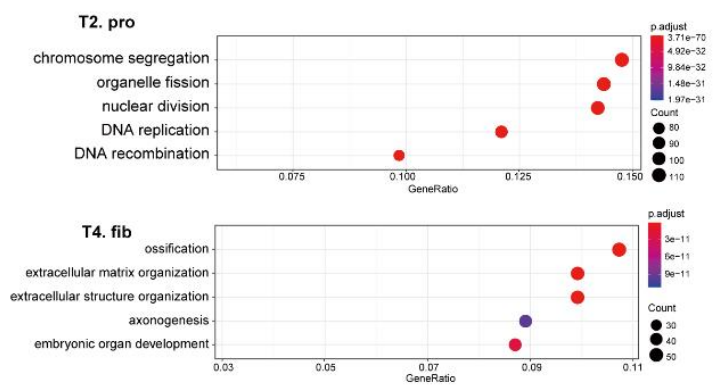

E
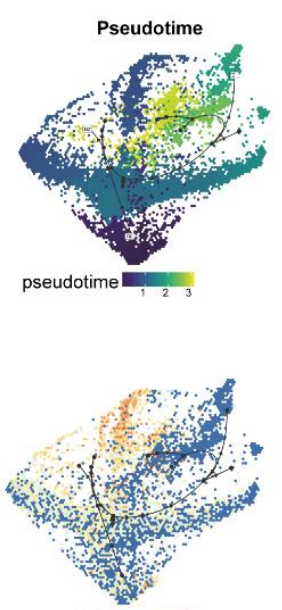

NOV

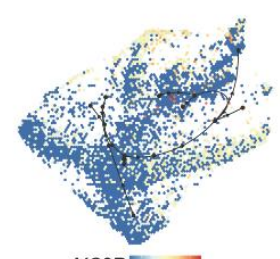

MC2R
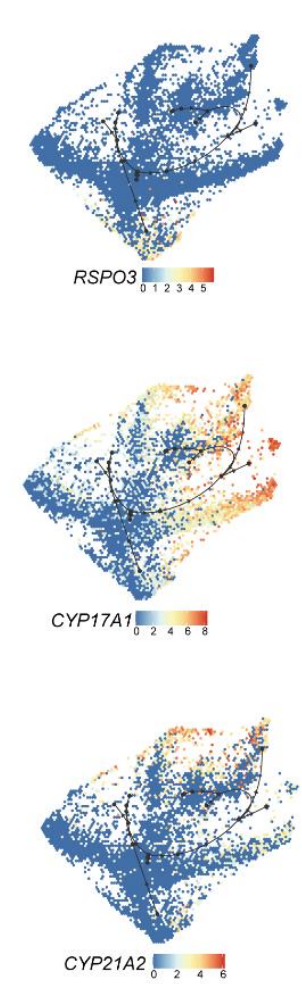

B

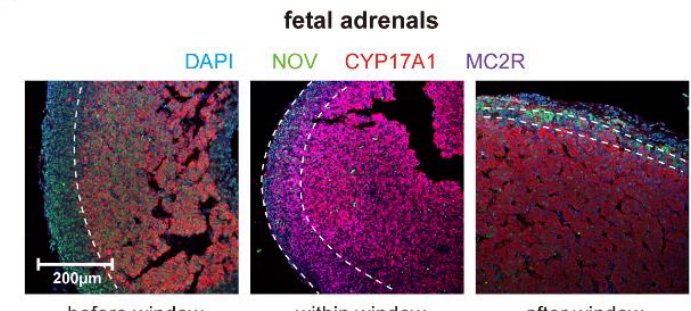

before window

within window

after window

D
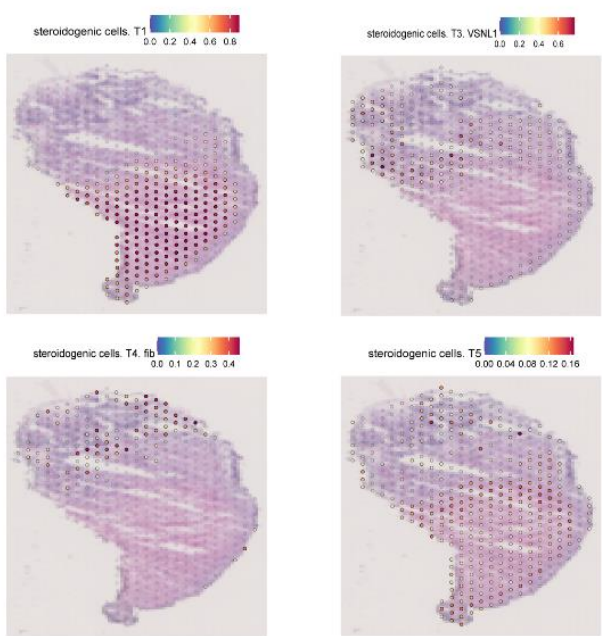

F
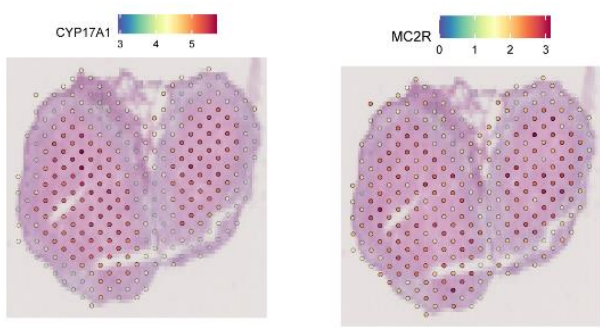

CYP21A2 1.01 .52 .02 .53 .0

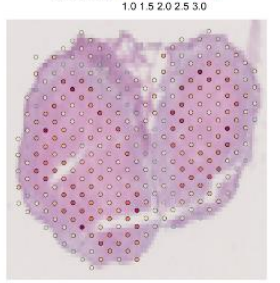

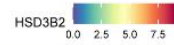

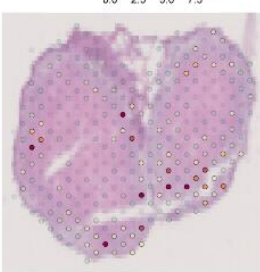

CYP1181

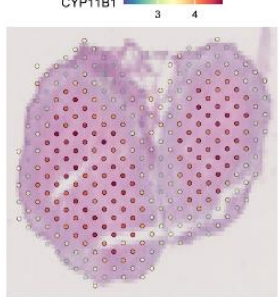

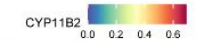

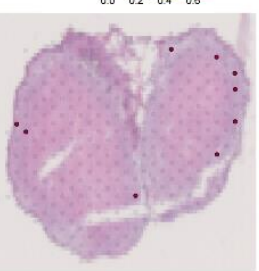

945 Figure EV1. Dynamic gene expression patterns of fetal adrenal steroidogenic cells. 
947 A. Uniform manifold approximation and projection analysis visualizations of the cell cycle score of professional steroidogenic cell populations. Cell cycle states: G1 (blue), G2/M (red), S (green).

B. Immunofluorescence staining of NOV (green), CYP17A1 (red), and MC2R (purple) in adrenal glands. Scale bar, $20 \mu \mathrm{m} . \mathrm{n}=3$.

C. Dot plot showing the GO functional analysis of T2 and T4 steroidogenic cluster cells. P value and percent of counts as in figure.

D. Visualization of the spatial transcriptome shows the location of T1, T3. VSNL1, T4. fib and T5 steroidogenic cells in 9GW fetal adrenal.

E. Pseudotime ordering of professional steroidogenic cells using Dyno. Expression of genes associated with the adrenal marker genes RSPO3, NOV, and $M C 2 R$ or the steroidogenic enzymes-related genes CYP17A1 and CYP21A2 mapped on professional steroidogenic cells. Color scale: red, high expression; blue, low expression.

F. Visualization of the spatial transcriptome shows the locations which high express adrenals. 
A

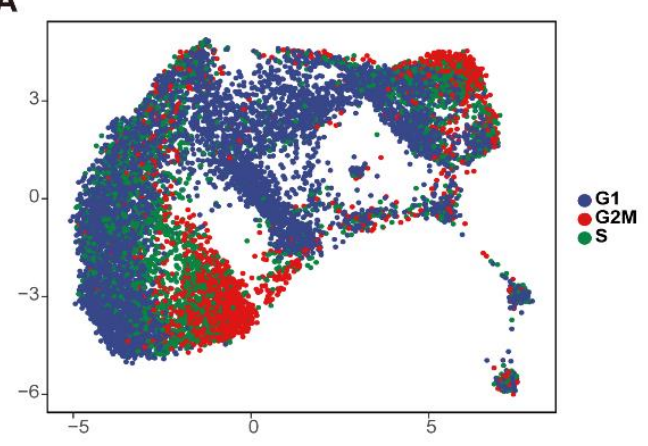

C

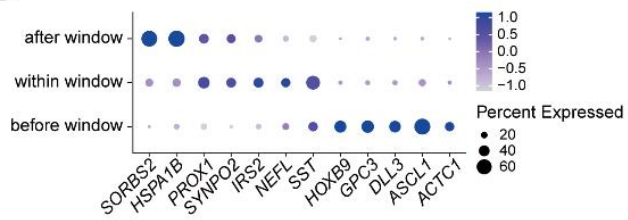

D

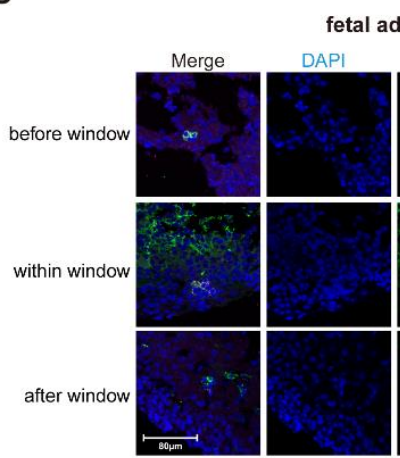

G

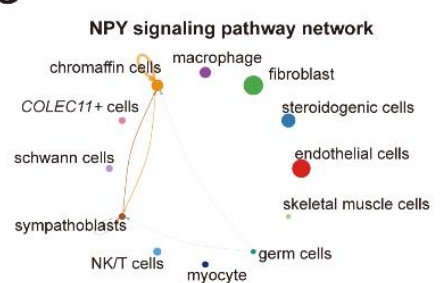

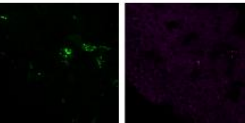

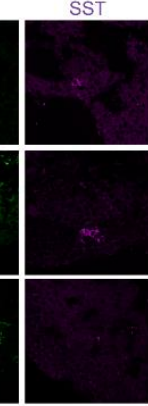

B
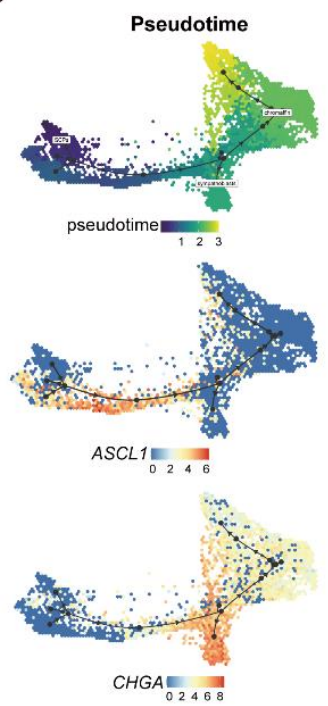

$F$

$\mathrm{E}$

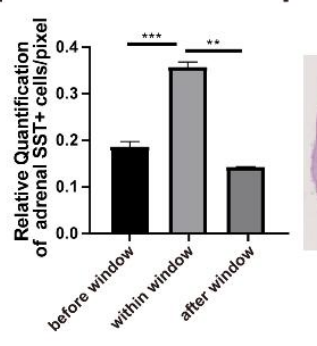

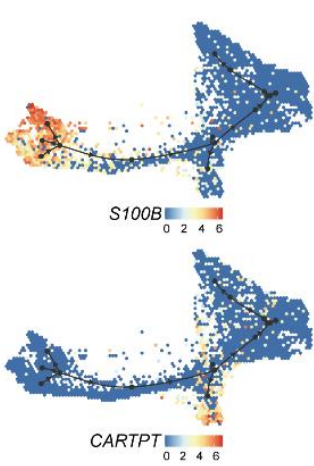
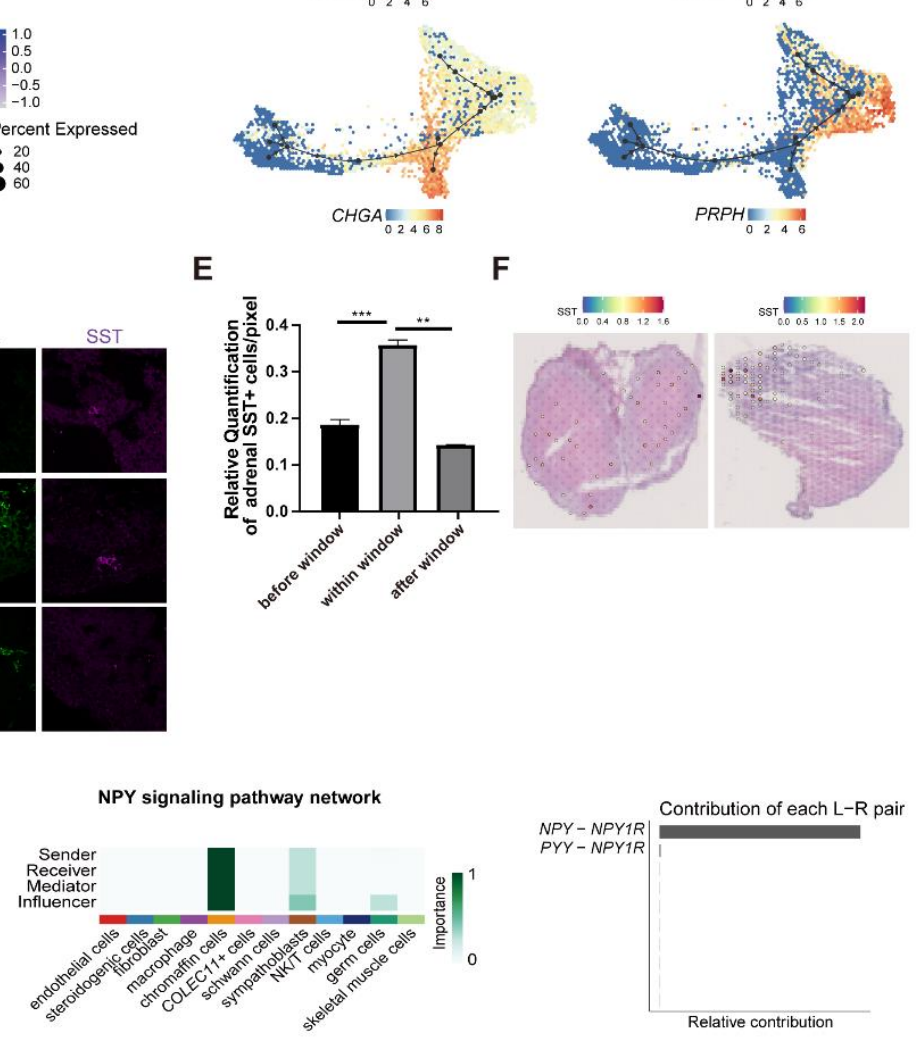

H

9GW fetal adrenals

Merge

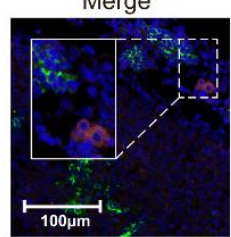
DAPI
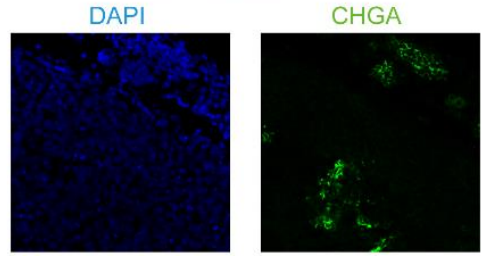

NPY

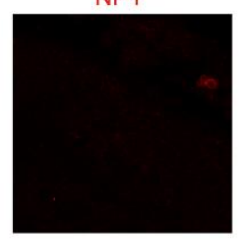

\section{Figure EV2. Transcriptional dynamics of human fetal adrenal neurocytes.}

967 A. Uniform manifold approximation and projection analysis visualizations of the cell cycle score of neurocyte populations. Cell cycle states: G1 (blue), G2/M (red), S (green). 
970 B. Pseudotime ordering of neurocyte populations using Dyno. Expression of genes associated with the indicated genes (S1OOB, ASCL1, CARTPT, CHGA, PRPH)

972 mapped. Color scale: red, high expression; blue, low expression.

973 C. Dot plots of differential gene expression of three stages of neurocyte groups

974 (before, within, and after sexual differentiation).

975 D. Immunofluorescence staining of SST (purple) and CHGA (green) in the adrenal 976 glands during the window of sexual differentiation.

977 E. Relative quantification numbers of adrenal SST+ neurocytes at different stages.

$978 \quad$ Scale bar, $20 \mu \mathrm{m} . \mathrm{n}=3$.

979 F. Visualization of the spatial transcriptome shows the locations which high express $980 \quad$ SST in $8 \mathrm{GW}$ fetal adrenals and 9GW fetal adrenal.

981 G. CellChat analysis showing the NPY cell-secreted signaling pathway among 982 chromaffin cells, sympathoblasts, and germ cells. Bar plot showing ligand983 receptor pair contributions mainly by NPY-NPY1R.

984 H. Immunofluorescence staining of NPY (red) and CHGA (green) in the adrenal 985 glands. Scale bar, $20 \mu \mathrm{m}$. 
A

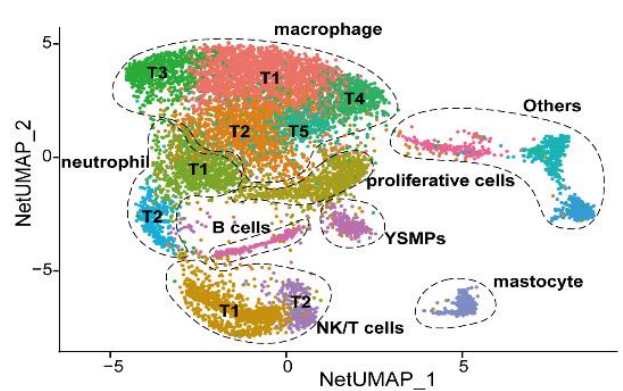

C

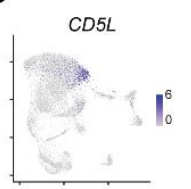

POMC

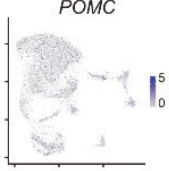

E

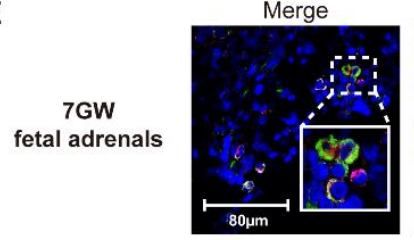

$\mathbf{F}$

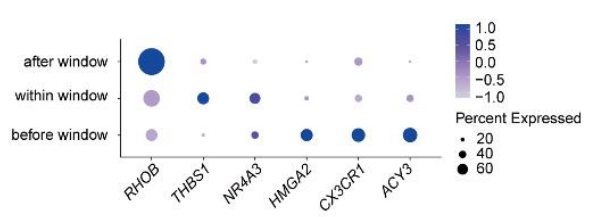

G

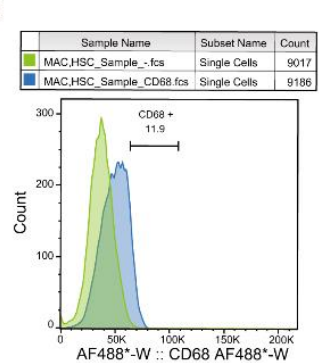

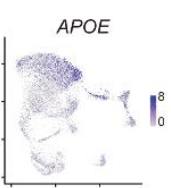

IGF1

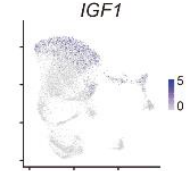

Merge $\mathrm{H}$

co-culture

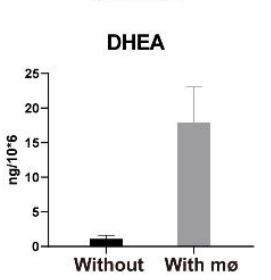

B

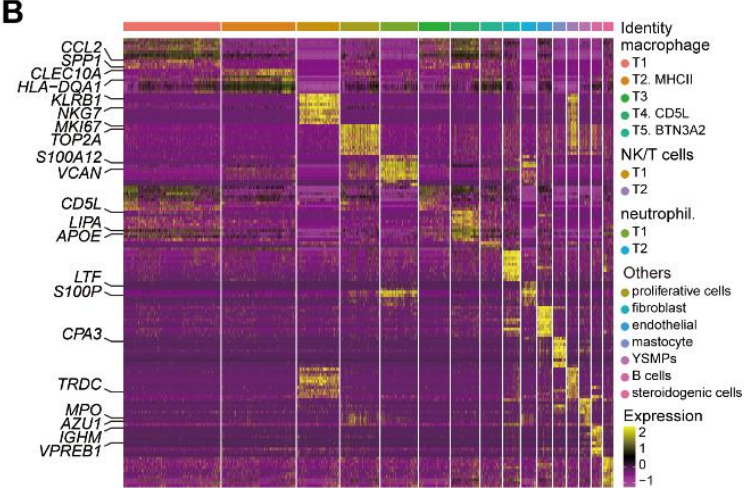

D

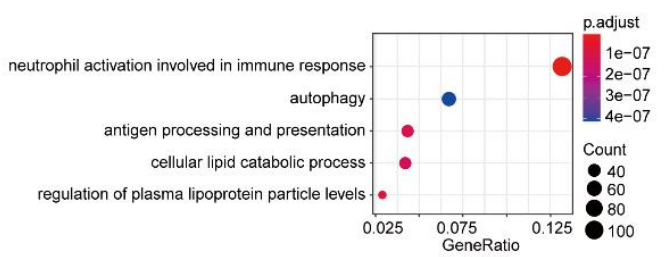

CD5L
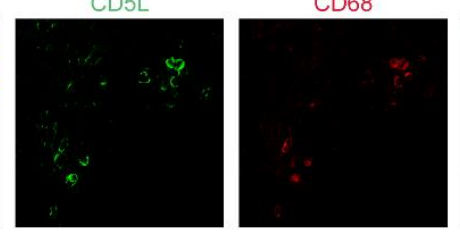

'

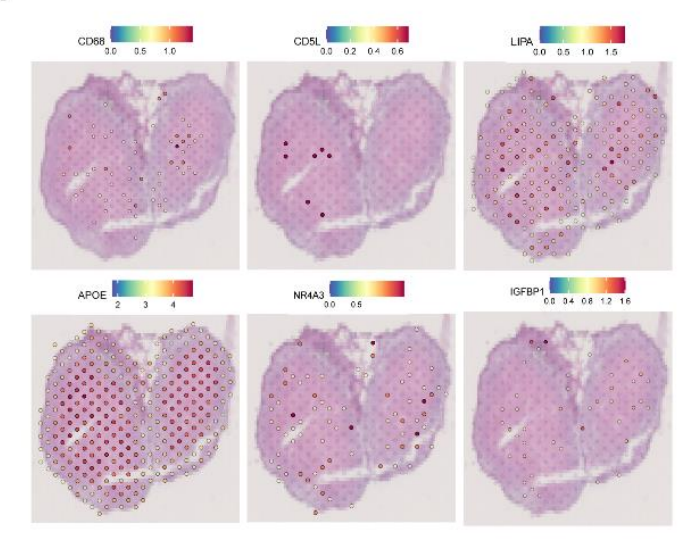

Figure EV3. A landscape and characteristics of fetal adrenal gland immune cells.

989 A. Uniform manifold approximation and projection analysis visualization of adrenal immune cells for $10 \times$ Genomics data $(n=10,704$ cells $)$.

991 B. Heat map of the top 10 differentially expressed genes (DEGs) between immune cell populations. Detailed cell information and DEGs can be found in Table EV5. 
993 C. Feature plot visualization of lipid metabolic regulators (CD5L, APOE, LIPA, $994 \quad P O M C, I G F 1$, and NR4A3) in fetal adrenal immune cell data.

995 D. Dot plot displaying the GO functional analysis of $C D 5 L+$ macrophages. $\mathrm{P}$ value 996 and percent of counts as in figure.

997 E. Immunofluorescence staining of CD5L (green), CD68 (red), and CYP17A1 998 (purple) in fetal adrenal tissues. Scale bar, $20 \mu \mathrm{m}$.

999 F. Dot plots of differential gene expression of three-stage immune cell groups 1000 (before, within, and after sexual differentiation).

1001 G. FACS analysis of CD68+ cells in fetal adrenal tissues at 8-12 gestational weeks $1002 \quad(\mathrm{n}=4)$.

1003 H. ELISA of DHEA in the supernatant of the in vitro cultured fetal adrenal cells, 1004 alone or cocultured with CD68+ (macrophage)-sorted cells from fetal adrenal 1005 tissues. mø, macrophage.

1006 I. Visualization of the spatial transcriptome shows the locations which high express CD68, CD5L, LIPA, APOE, NR4A3 and IGFBP1 in 8GW fetal adrenals. 
A

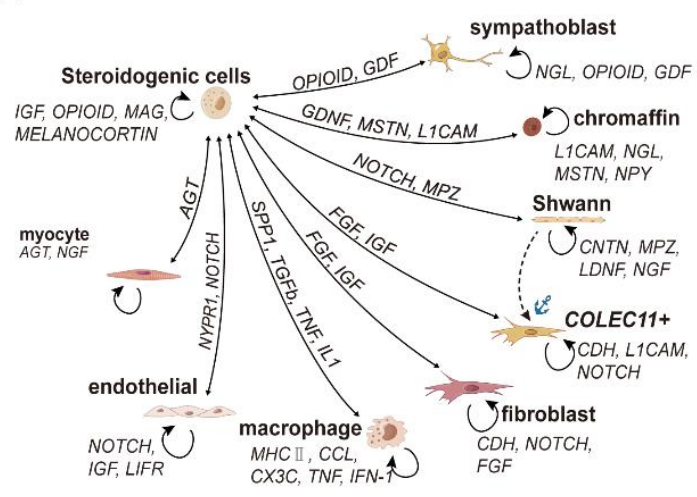

B

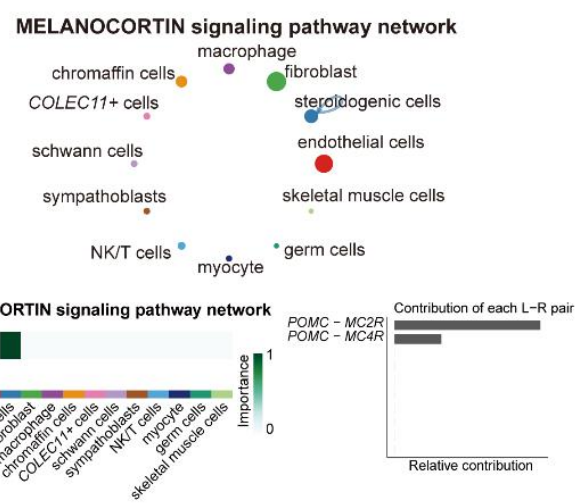

D

C
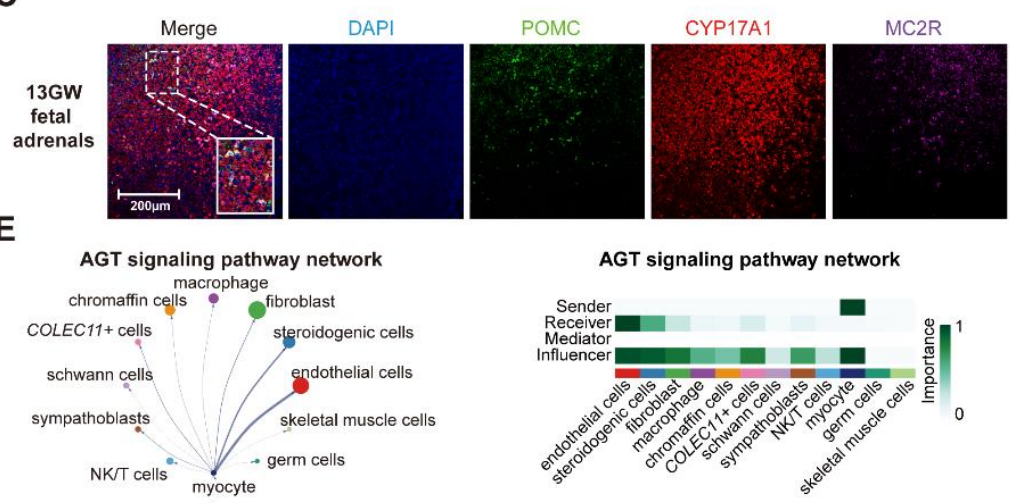

AGT signaling pathway network
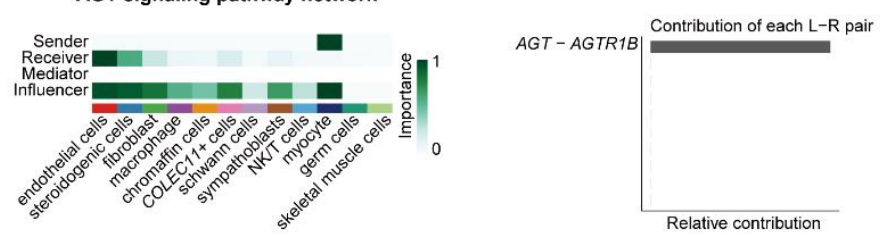

$\mathbf{F}$
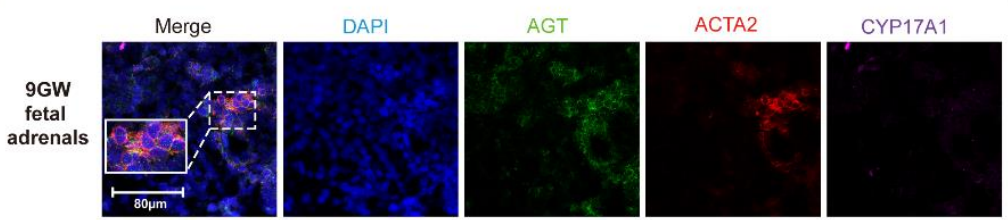

G

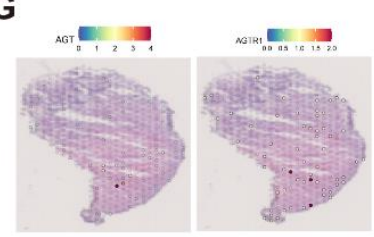

H
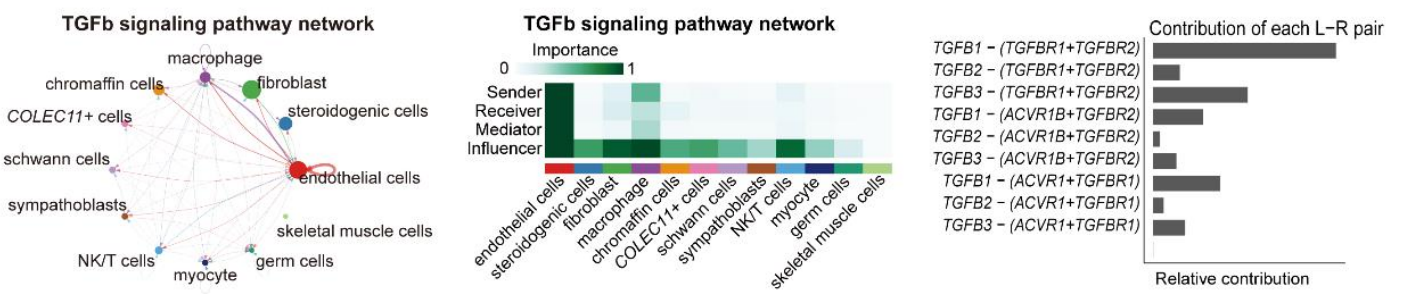

I
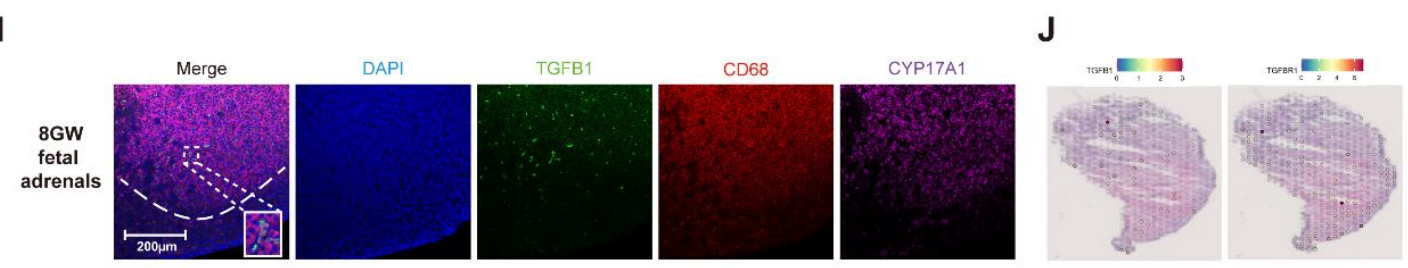

Figure EV4. Cellular interactions in fetal adrenal glands.

1011 A. Diagram of the regulatory network between professional steroidogenic cells and other cells in the fetal adrenal glands. 
B. CellChat analysis showing the melanocortin-secreted signaling pathway within the professional steroidogenic cell cluster itself. Bar plot showing ligand-receptor pair contributions by $P O M C-M C 2 R$ and $P O M C-M C 4 R$.

C. Immunofluorescence staining of POMC (green), MC2R (purple), and CYP17A1 (red) in adrenal glands at 13 gestational weeks (GW). Scale bar, $20 \mu \mathrm{m}$.

D. Visualization of the spatial transcriptome shows the locations which high express $P O M C$ and $M C 2 R$ in $9 \mathrm{GW}$ fetal adrenal.

E. CellChat analysis showing the AGT-secreted signaling pathway between myocytes and other types of cells. Bar plot showing ligand-receptor pair contribution by $A G T-A G T R$.

F. Immunofluorescence staining of AGT (green), ACTA2 (red), and CYP17A1 (purple) in adrenal glands at $9 \mathrm{GW}$. Scale bar, $20 \mu \mathrm{m}$.

G. Visualization of the spatial transcriptome shows the locations which high express $A G T$ and $A G T R 1$ in $9 \mathrm{GW}$ fetal adrenal.

H. CellChat analysis showing the growth regulator TGFb cell-secreted signaling pathway between immune cells and other types of cells. Bar plot showing ligandreceptor pair contributions mainly by TGFB1-(TGFBR1+TGFBR2).

I. Immunofluorescence staining of TGFB1 (green), CD68 (red), and CYP17A1 (purple) in the adrenal glands. Scale bar, $20 \mu \mathrm{m}$.

J. Visualization of the spatial transcriptome shows the locations which high express TGFB1 and TGFBR1 in 9GW fetal adrenal. 
A

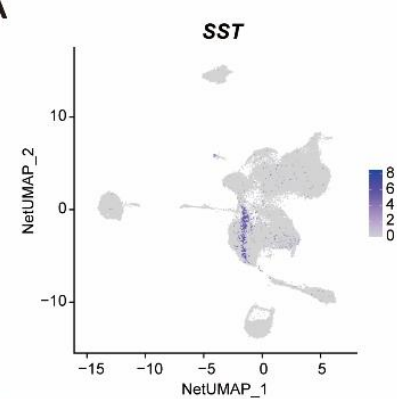

C
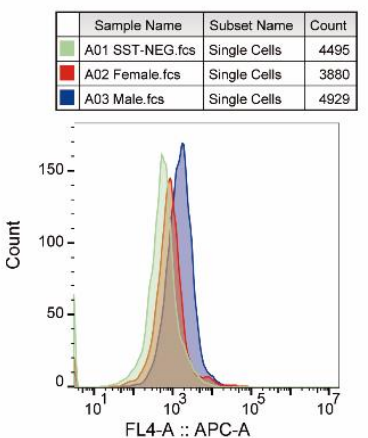

E

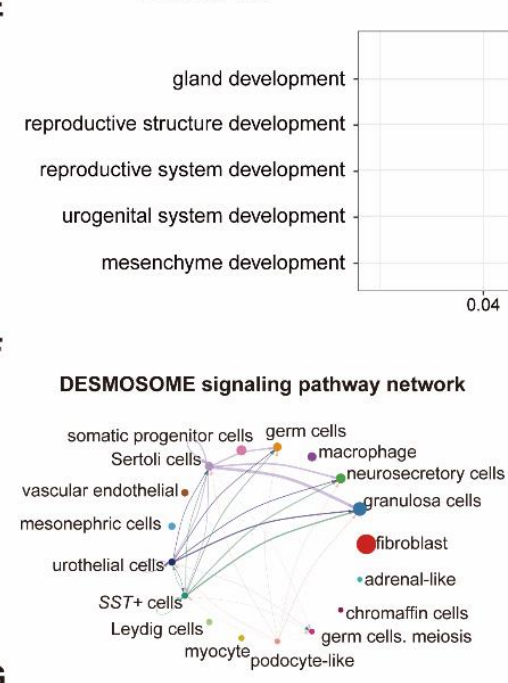

G
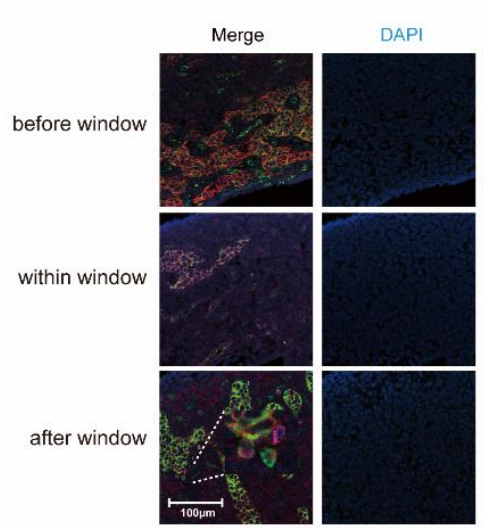

B
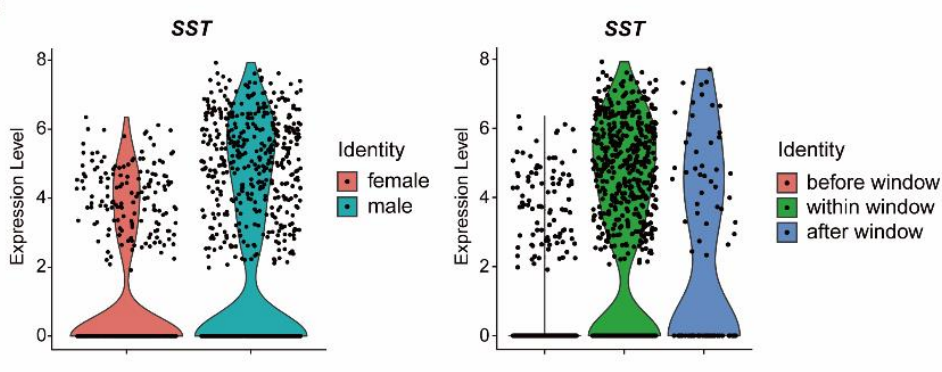

D
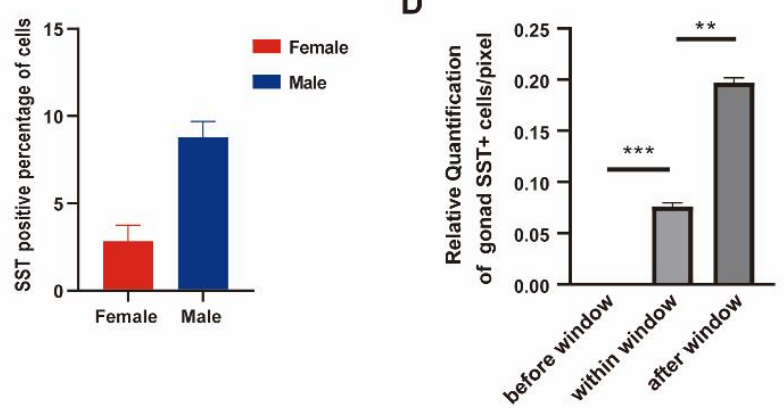
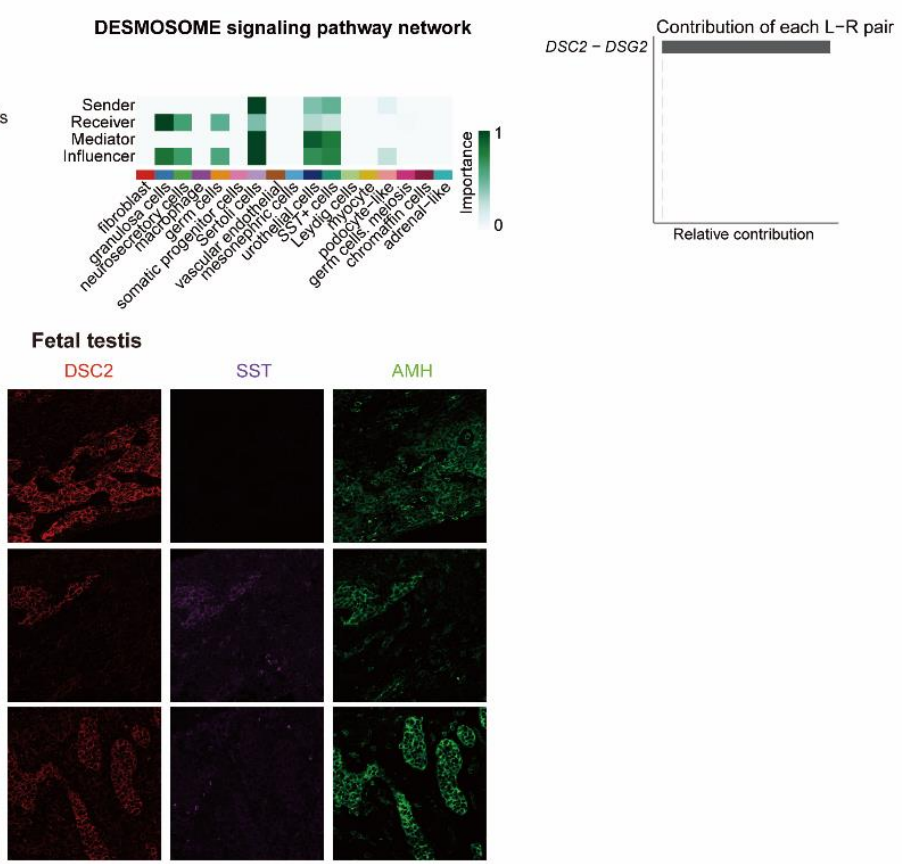

Figure EV5. Characteristics of SST+ cells in fetal gonads.

A. Featureplot showing the cluster of SST+ cells in the gonadal $10 \times$ Genomics data. 
B. Violin plots of SST gene expression patterns of female and male gonads spanning the window of sexual differentiation.

C. FACS analysis of fetal gonad SST+ cells at 8-10 gestational weeks. Male n = 3,

1040 female $\mathrm{n}=3$.

1041 D. Relative quantification numbers of gonadal SST+ cells at different stages.

E. Dot plot showing the GO function analysis of SST+ cells. P value and percent of counts as in figure.

1044 F. CellChat analysis showing the desmosome cell-cell contact signaling pathway between SST+ cells and other gonadal somatic cells. Bar plot showing ligandreceptor pair contributions by DSC2-DSG2.

1047 G. Immunofluorescence staining of AMH (green), DSC2 (red), and SST (purple) in testes spanning the window of sexual differentiation. Scale bar, $20 \mu \mathrm{m} . \mathrm{n}=3$. 


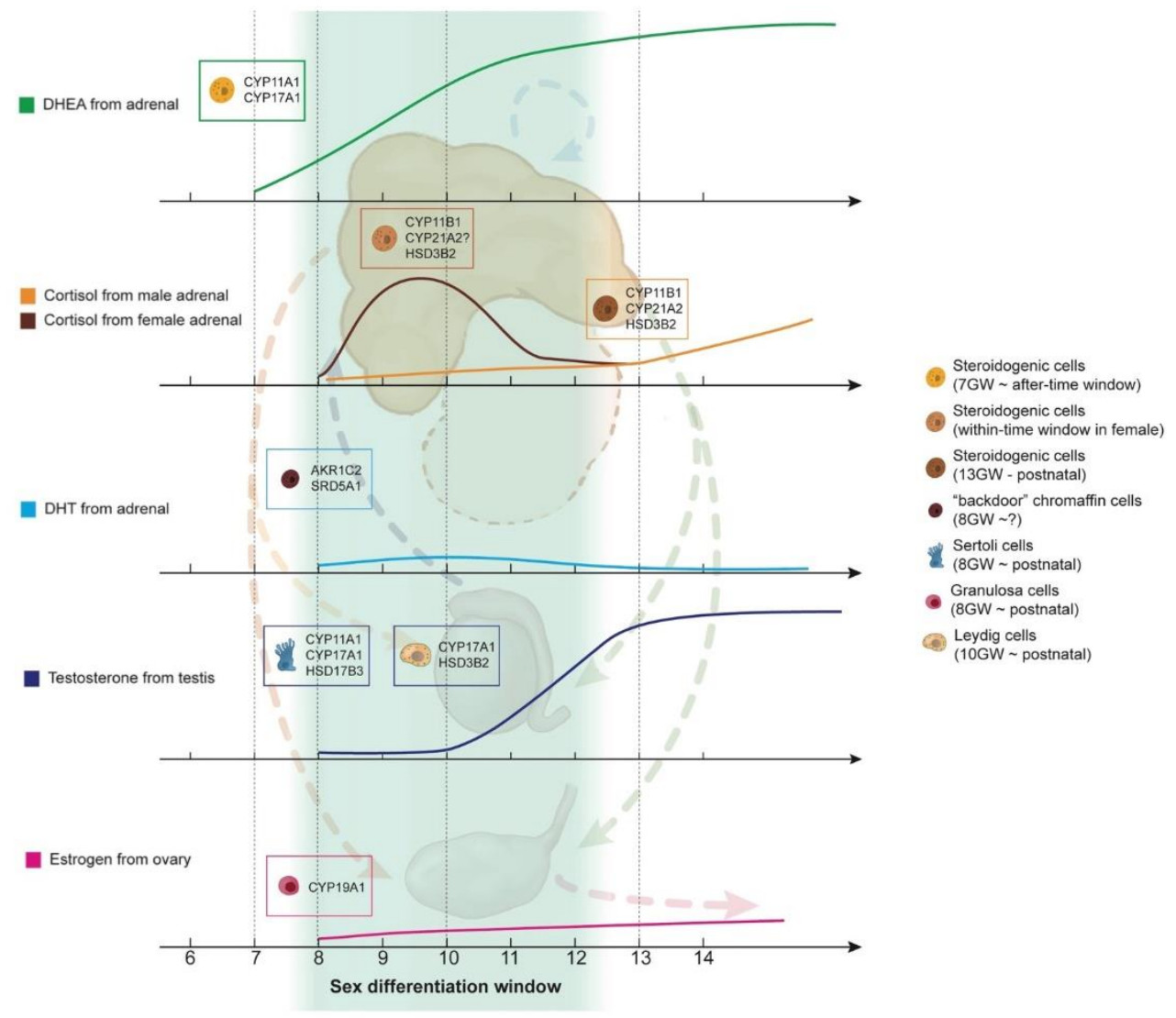

1051 Figure EV6. A schematic illustration showing cell-type-specific steroidogenic-

1052 related genes and steroid levels in adrenal glands and gonads. 